\title{
UNRAMIFIED COHOMOLOGY OF QUADRICS, II
}

\author{
BRUNO KAHN AND R. SUJATHA
}

\section{Contents}

1. Introduction 1

1.1. The sequence (1) 2

1.2. $\operatorname{Ker} \eta_{2}^{4} \quad 3$

1.3. Coker $\eta_{2}^{4}$ and Coker $\eta^{4} \quad 3$

2. Proof of theorem 2

3. Coker $\eta^{4}$ : some preparations. Proof of theorem $3 \quad 8$

3.1. The cases $\operatorname{dim} X=2,3$; proof of theorem $3 \quad 8$

3.2. The case $\operatorname{dim} X=4 \quad 9$

4. Proof of theorem 4 10

4.1. Generalities 10

4.2. The neighbour case 11

4.3. The intermediate case 11

4.4. The Albert case 13

4.5. The virtual Albert case 15

5. Proofs of theorems 1 and $5 \quad 22$

5.1. Proof of theorem $1 \quad 22$

5.2. Proof of theorem 5

Appendix A. A spectral sequence for the étale motivic cohomology of an $\begin{array}{ll}\text { affine quadric } & 27\end{array}$

$\begin{array}{ll}\text { References } & 28\end{array}$

\section{INTRODUCTION}

In this paper, we continue our investigation of the unramified cohomology of quadrics, initiated in [10]. Let $F$ be a field of characteristic $\neq 2$. For any $F$-scheme $X$, let $H^{i} X$ denote the étale cohomology group $H_{\text {ét }}^{i}(X, \mathbf{Z} / 2)$ and $H^{i}(X, i-1)$ the group $H_{\text {ét }}^{i}(X, \mathbf{Q} / \mathbf{Z}(i-1))$, where $\mathbf{Q} / \mathbf{Z}(i-1)=\underline{\lim }_{(n, \operatorname{char} F)=1} \mu_{n}^{\otimes i-1}$. We recall from loc. cit. the unramified cohomology groups

$$
\begin{gathered}
H_{\mathrm{nr}}^{i}(F(X) / F)=\operatorname{Ker}\left(H^{i} F(X) \rightarrow \coprod_{x \in X^{(1)}} H^{i-1} F(x)\right) \\
H_{\mathrm{nr}}^{i}(F(X) / F, i-1)=\operatorname{Ker}\left(H^{i} F(X, i-1) \rightarrow \coprod_{x \in X^{(1)}} H^{i-1}(F(x), i-2)\right.
\end{gathered}
$$


for a smooth, proper, geometrically integral variety $X$ over $F$. Extension of scalars from $F$ to $F(X)$, the function field of $X$, determines homomorphisms

$$
\begin{aligned}
& \eta_{2}^{i}: H^{i} F \rightarrow H_{\mathrm{nr}}^{i}(F(X) / F) \\
& \eta^{i}: H^{i}(F, i-1) \rightarrow H_{\mathrm{nr}}^{i}(F(X) / F, i-1) .
\end{aligned}
$$

We are interested in the kernel and cokernel of $\eta_{2}^{i}$ and $\eta^{i}$ when $X$ is a quadric. If $X$ is the quadric defined by a quadratic form $q$, we sometimes write $\eta_{2, q}^{i}$ for precision. We then have $\operatorname{dim} X=\operatorname{dim} q-2$. For the reader's convenience, let us briefly recall the results of [10]:

a) For any quadric $X$ and any $i, \operatorname{Ker} \eta_{2}^{i} \stackrel{\sim}{\rightarrow}$ Ker $\eta^{i}$. For $i \leq 4$, there is an exact sequence

$$
0 \rightarrow\left(\operatorname{Ker} \eta_{2}^{i}\right)_{0} \rightarrow \text { Coker } \eta_{2}^{i} \rightarrow \text { Coker } \eta^{i}
$$

where

$$
\left(\operatorname{Ker} \eta_{2}^{i}\right)_{0}=\left\{\alpha \in \operatorname{Ker} \eta_{2}^{i} \mid \alpha \cdot(-1)=0\right\} .
$$

Let us mention here that the construction of this exact sequence relies on the Milnor conjecture. From the proof of this conjecture by Voevodsky, it follows that (1) is in valid for any $i$. (Compare [10, proof of prop. 3].)

b) For $i \leq 4$ and $\operatorname{dim} X>2^{i-2}-2$, Ker $\eta_{2}^{i}$ consists of symbols, i.e. of elements of the form $\left(a_{1}, \ldots, a_{i}\right)$ for $a_{1}, \ldots, a_{i} \in F^{*}$. A symbol $\left(a_{1}, \ldots, a_{i}\right)$ lies in Ker $\eta_{2, q}^{i}$ if and only if $q$ is similar to a subform of the Pfister form $\ll a_{1}, \ldots, a_{i} \gg$. In particular, it is

- 0 for $\operatorname{dim} X>2^{i}-2$

- at most $\mathbf{Z} / 2$ for $\operatorname{dim} X>2^{i-1}-2$.

Although we feel that the above must remain true for any $i$, it is not a straightforward consequence of the Milnor conjecture and we don't know a proof of it, say, for $i=5$.

c) For $i \leq 2$, Coker $\eta^{i}=0$ for all quadrics. The group Coker $\eta^{3}$ is 0 , except when $X$ is an anisotropic Albert quadric (see subsection 1.3 for a definition), in which case this group is isomorphic to $\mathbf{Z} / 2$. Then the map Coker $\eta_{2}^{3} \rightarrow$ Coker $\eta^{3}$ is bijective. As for Coker $\eta^{4}$, our main results are as follows: this group is 0 for $\operatorname{dim} X=1$ or $\operatorname{dim} X>10$. For $\operatorname{dim} X>4$, it embeds canonically into the 2-torsion of $C H^{3}(X)$, which is itself at most $\mathbf{Z} / 2$ (Karpenko).

Here we shall deal mostly with the cases $i=4$, $\operatorname{dim} X=2,3,4$. The case where $i=4,5 \leq \operatorname{dim} X \leq 10$ will be dealt with in another paper [11]. This paper will also contain the results on real quadrics announced in [10].

Let us now describe the results of the current paper.

1.1. The sequence (1). A vexing issue is whether the map Coker $\eta_{2}^{i} \rightarrow$ Coker $\eta^{i}$ is always surjective. We can prove:

Theorem 1. Suppose that F contains all 2-primary roots of unity. Then, for any quadric and any $i \geq 0$, the map Coker $\eta_{2}^{i} \rightarrow$ Coker $\eta^{i}$ is surjective. 
It seems quite difficult to make a descent from theorem 1, even in the case where $F$ contains a 4 th root of unity. The minimal possible counterexample to surjectivity in general is in the case $i=4, X$ a virtual Albert quadric (see subsection 1.3 for a definition). To the best of our efforts, we have not been able to decide what happens in this case. At least we are able to prove surjectivity if we assume in addition that the 2-cohomological dimension of $F$ is $\leq 4$. See theorem 5 below.

1.2. $\operatorname{Ker} \eta_{2}^{4}$. Let us say that a subgroup $A$ of $H^{i} F$ is generated by its symbols if every element of $A$ is a sum of symbols, each of which belongs to $A$.

Obviously, $A$ is generated by its symbols if it consists of symbols. The results recalled above then imply that Ker $\eta_{2}^{i}$ is generated by its symbols for $i \leq 4$ and $\operatorname{dim} X>2^{i-2}-2$. For $i \leq 3$, this covers all quadrics, and for $i=4$, it covers all quadrics of dimension $>2$. The following theorem deals with the remaining cases for $i=4$.

Theorem 2. For any quadric $X$ of dimension $\leq 2$ over $F$,

a) $\operatorname{Ker} \eta_{2}^{4}=H^{1} F \cdot \operatorname{Ker} \eta_{2}^{3}$.

b) Ker $\eta_{2}^{4}$ is generated by its symbols.

To prove theorem 2, we use the results of [16]. We note that, amusingly, the computation of $\operatorname{Ker} \eta_{2}^{4}$ for a 3-dimensional Pfister neighbour, which is the main result of [5] and [23], can be easily deduced from [16, (9.2)] and our theorem 2.1 below. We also provide an alternate proof of theorem 2 by using the results of [9] (see remark 2.7).

Note the following corollary to theorem 2 and the results recalled before it (compare $[4$, th. 3.2]):

Corollary 1. Let $i \leq 4, n \geq 0$ and $q, \varphi$ be respectively a quadratic form and an $n$-fold Pfister form over $F$. Then

$$
e^{n}(\varphi) \operatorname{Ker} \eta_{2, q}^{i} \subseteq \operatorname{Ker} \eta_{2, \varphi \otimes q}^{n+i} .
$$

Proof. We argue as in [4]. We have to prove that, for $x \in \operatorname{Ker} \eta_{2, q}^{i}, e^{n}(\varphi) \cdot x \in$ $\operatorname{Ker} \eta_{2, \varphi \otimes q}^{n+i}$. By theorem 2, we may assume that $x$ is a symbol, say $x=e^{i}(\tau)$ for an $i$-fold Pfister form $\tau$. Since $x_{F(q)}=0$, we have $\tau_{F(q)} \sim 0$ [20], hence $q$ is similar to a subform of $\tau$ [1, Satz 1.3]. Then $\varphi \otimes q$ is similar to a subform of the $(n+i)$-fold Pfister form $\varphi \otimes \tau$. Therefore $(\varphi \otimes \tau)_{F(\varphi \otimes q)} \sim 0$ and $e^{n+i}(\varphi \otimes \tau)_{F(\varphi \otimes q)}=\left(e^{n}(\varphi) \cdot x\right)_{F(\varphi \otimes q)}=0$.

1.3. Coker $\eta_{2}^{4}$ and Coker $\eta^{4}$. Here we need to assume that char $F=0$. We have the following results.

Theorem 3. Let $X$ be a quadric of dimension 2 or 3. Then Coker $\eta^{4}=0$.

By [10, prop. A.1 and A.2], the same result holds for $\operatorname{dim} X=1$ or 0 (the latter with a suitable definition of Coker $\eta^{4}$ ).

Assume now that $\operatorname{dim} X=4$. For the reader's convenience, we recall the classification of (anisotropic) 4-dimensional quadrics used in [10]. Let $X$ be such a quadric, $q$ be a quadratic form defining it and $d=d_{ \pm} q \in F^{*} / F^{* 2}$. 
- Neighbours: $d \neq 1, q_{F(\sqrt{d})}$ is hyperbolic. (These are the 6-dimensional qs that are Pfister neighbours.)

- Intermediate quadrics: $d \neq 1, q_{F(\sqrt{d})}$ is isotropic without being hyperbolic. (These forms did not receive a name in [10].)

- (Anisotropic) Albert quadrics: $d=1$.

- Virtual Albert quadrics: $d \neq 1, q_{F(\sqrt{d})}$ is anisotropic.

Theorem 4. Let $X$ be a 4-dimensional quadric.

a) If $X$ is neighbour or intermediate, then Coker $\eta^{4}=0$.

b) If $X$ is an Albert quadric, then cup-product by the generator e of Coker $\eta^{3}$ gives an isomorphism

$$
F^{*} / \operatorname{Sn}(X) \stackrel{\sim}{\longrightarrow} \text { Coker } \eta^{4} .
$$

Here $\operatorname{Sn}(X)$ is the group of spinor norms of $X$, i.e. the subgroup of $F^{*}$ generated by products of two nonzero values of $q$, where $q$ is any quadratic form defining $X$.

c) If $X$ is a virtual Albert quadric of discriminant d, let $E=F(\sqrt{d})$. Then there is an exact sequence

$$
\text { Coker } \eta_{E}^{4} \stackrel{\mathrm{Cor}_{E / F}}{\longrightarrow} \text { Coker } \eta^{4} \rightarrow P S O(q, F) / R \rightarrow 0
$$

where Coker $\eta_{E}^{4}=$ Coker $\eta_{X_{E}}^{4}$ and $P S O(q, F) / R$ is the group of rational points of the projective special orthogonal group of $q$, modulo $R$-equivalence. Here $q$ is any quadratic form defining $X$.

In [19], Merkurjev shows that if $q$ is a quadratic form of dimension $\leq 6$, the group $P S O(q)$ is not $R$-trivial if and only if $q$ is a virtual Albert form. It is therefore striking to see the group $P S O(q, F) / R$ appear as a quotient of Coker $\eta^{4}$ in the latter case. This is the first known cohomological description of this group.

Corollary 2. In theorem 4, the map Coker $\eta_{2}^{4} \rightarrow$ Coker $\eta^{4}$ is surjective, except perhaps in the case of a virtual Albert quadric.

Proof. Indeed, the only issue is that of an Albert quadric. By [10], Coker $\eta_{2}^{3} \rightarrow$ Coker $\eta^{3}$ is then bijective, so cup-product by the element $e$ of b) factors through Coker $\eta_{2}^{3}$.

We haven't computed the kernel of the map $F^{*} \rightarrow$ Coker $\eta_{2}^{4}$ in theorem $4 \mathrm{~b}$ ); this would be an interesting exercise.

Theorem 5. Let $X$ be a virtual Albert quadric. Then the cokernel of the map

$$
\text { Coker } \eta_{2}^{4} \rightarrow \text { Coker } \eta^{4}
$$

is a subgroup of Ker $\eta_{2}^{5} /(-1) \cdot \operatorname{Ker} \eta_{2}^{4}$. In particular, it is 0 if $c d_{2}(F)<5$.

For the proof of theorems 3,4 and 5, we use the results of [9]. This the only reason why we need to assume that $\operatorname{char} F=0$, as the spectral sequences of $[9]$ rely on results of Voevodsky which require resolution of singularities. Although he declined to be a coauthor of this paper in the end, it is Markus Rost who first suggested that theorem 3 and theorem 4 b) should hold. He also explained the proof 
of theorem 3 that we give here, assuming the existence of spectral sequences as in $[9]$.

The structure of this paper is as follows. Theorem 2 is proven in section 2. Theorem 3 is proven in section 3, which also contains material for the next section. Theorem 4 is proven in section 4; the most daunting case is that of a virtual Albert quadric, which alone occupies 7 of the 13 pages in this section! Theorems 1 and 5 are proven in section 5. Finally, we construct in the appendix a spectral sequence analogous to those of [9] for an affine quadric: this spectral sequence is used in section 4 for the virtual Albert case.

Acknowledgements. This work was performed at the occasion of several visits of the first author to TIFR, Mumbai; he wishes to express his thanks to this institute for its excellent working conditions and stimulating environment. The second author wishes to thank the Alexander von Humboldt Stiftung for support and Universität Regensburg for hospitality, when the work was in progress.

\section{Proof of TheOREM 2}

Let $X$ be a smooth, projective quadric of dimension $\geq 1$. Choose a conic $C$ traced over $X$ and let $D$ be the quaternion algebra associated with $C$ : if $C$ is defined by a 3-dimensional form $q$, then $D=C_{0}(q)$. Recall from [16] the algebraic $K$-theory $K_{*}(X, D)$ of $X$ with operators $D$ (denoted there by $\left.K_{*}^{D}(X)\right)$ : this is the $K$-theory of the exact category of locally free $\mathcal{O}_{X}$-modules provided with a left action of $D$. We have a Brown-Gersten-Quillen spectral sequence

$$
E_{2}^{p, q}=H^{p}\left(X, \mathcal{K}_{-q}^{D}\right) \Rightarrow K_{-p-q}(X, D)
$$

where $H^{p}\left(X, \mathcal{K}_{-q}^{D}\right)$ denotes the $p$-th cohomology group of an appropriate Gersten complex. We also have a Swan-style isomorphism

$$
K_{*}(D)^{n} \oplus K_{*}\left(C_{0} \otimes_{F} D\right) \stackrel{\sim}{\rightarrow} K_{*}(X, D)
$$

described in $[16,(4.1)]$ (here $n=\operatorname{dim} X$ and $C_{0}=C_{0}(q)$, where $q$ is any quadratic form defining $X)$.

2.1. Theorem. There is a natural isomorphism

$$
\operatorname{Ker} \eta_{2}^{4} \stackrel{\sim}{\rightarrow} \frac{H^{0}\left(X, \mathcal{K}_{2}^{D}\right)}{K_{2} D}
$$


Proof. Consider the following commutative diagram

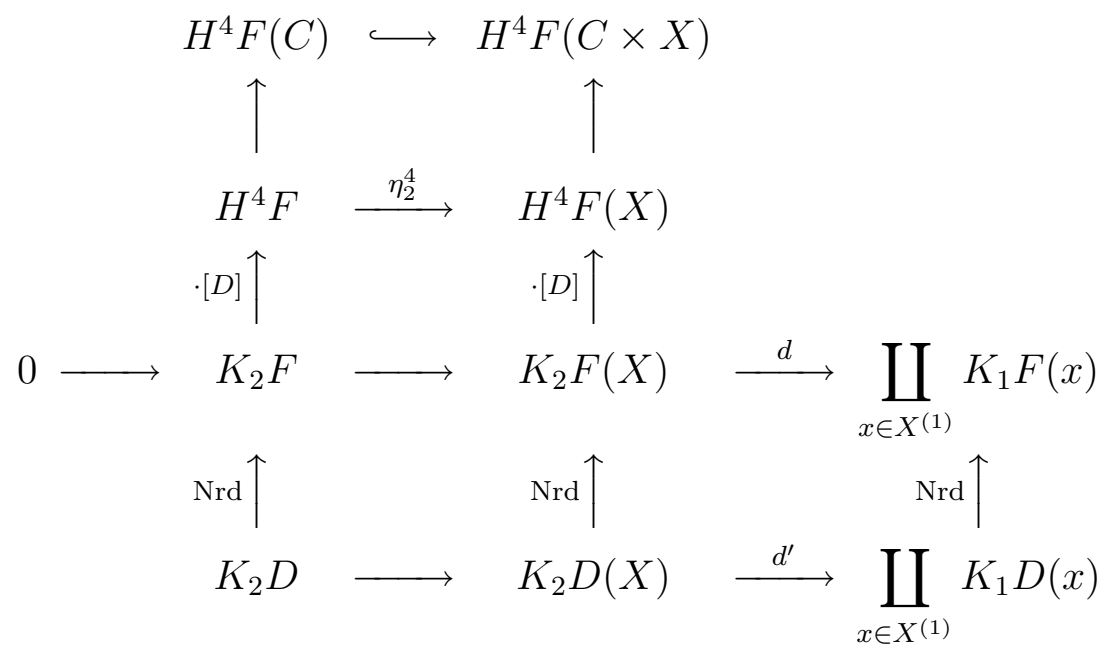

In this diagram, both rows are complexes and the maps labeled $d$ and $d^{\prime}$ have (by definition) respective kernels $H^{0}\left(X, \mathcal{K}_{2}\right)$ and $H^{0}\left(X, \mathcal{K}_{2}^{D}\right)$. The top horizontal map is injective because $X$ is isotropic over $F(C)$. The columns are exact at $H^{4} F$ and $H^{4} F(X)$ by the one-dimensional case, and at $K_{2} F$ and $K_{2} F(X)$ by [16, th. 2]. The bottom right square commutes by $[16,(3.14)]$. Finally, the reduced norm on $K_{1}$ of quaternion algebras is injective by Wang's theorem, so that the bottom right vertical arrow is injective. These remarks and an easy diagram chase give the map we want.

The third row of the above diagram is exact: at $K_{2} F$ it follows from [22, th. 3.6] and at $K_{2} F(X)$ it follows from [22, cor 5.6], since $X$ is a complete rational variety. This implies that our map is an isomorphism.

2.2. Proposition. $[16, \S 11]$ Let $n=\operatorname{dim} X$. Then:

a) The norm homomorphism $H^{n}\left(X, \mathcal{K}_{n+1}^{D}\right) \stackrel{N}{\longrightarrow} K_{1} D$ is an isomorphism.

b) The natural map $H^{n}\left(X, \mathcal{K}_{n+1}^{D}\right) \rightarrow K_{1}(X, D)^{(n)}$ is an isomorphism.

In particular, all differentials arriving at $H^{n}\left(X, \mathcal{K}_{n+1}^{D}\right)$ in the spectral sequence (2) are 0 .

2.3. Corollary. If $n=2$, the map $K_{2}(X, D) \rightarrow H^{0}\left(X, \mathcal{K}_{2}^{D}\right)$ is surjective. Therefore there is a canonical isomorphism

$$
\frac{K_{2}(X, D)^{(0 / 1)}}{K_{2} D} \stackrel{\sim}{\rightarrow} \frac{H^{0}\left(X, \mathcal{K}_{2}^{D}\right)}{K_{2} D} .
$$

Suppose $\operatorname{dim} X=2$; let $d=d_{ \pm} X$ and $E=F(\sqrt{d})$. Then $C_{0} \simeq D \otimes_{F} E$, hence $C_{0} \otimes_{F} D$ is a simple algebra similar to $E$. On the other hand, $X$ is hyperbolic on $E(X)$, hence $E(X)$ splits $D(X):=D \otimes_{F} F(X)$ and so is contained in $D(X)$. This allows us to define a map $\nu: K_{*} E \rightarrow K_{*} D(X)$ as the composite

$$
K_{*} E \rightarrow K_{*} E(X) \rightarrow K_{*} D(X) .
$$


2.4. Proposition. The composite

$$
K_{*} D \oplus K_{*} D \oplus K_{*} E \stackrel{\sim}{\longrightarrow} K_{*}(X, D) \rightarrow K_{*} D(X)
$$

maps a triple $(f, g, v)$ to $f_{D(X)}+g_{D(X)}+\nu(v)$. Here the first map is Swan's isomorphism (3).

Proof. This follows from an elementary computation in the spirit of $[16,(4.3)$ and (4.11)].

2.5. Corollary. The composition

$$
K_{2} E \hookrightarrow K_{2}(X, D) \rightarrow K_{2}(X, D)^{(0 / 1)} \rightarrow \frac{K_{2}(X, D)^{(0 / 1)}}{K_{2} D}
$$

is surjective, where the first map is given by Swan's isomorphism.

2.6. Proposition. Let $X$ be a 2-dimensional quadric defined by a quadratic form of discriminant $d \neq 1$ and $E=F(\sqrt{d})$. Then the corestriction map

$$
\operatorname{Cor}_{E / F}: \operatorname{Ker}\left(H^{4} E \rightarrow H^{4} E(X)\right) \rightarrow \operatorname{Ker}\left(H^{4} F \rightarrow H^{4} F(X)\right)
$$

is surjective.

Proof. By theorem 2.1, corollary 2.3 and corollary 2.5, it suffices to prove that the map

$$
K_{2}\left(E \otimes_{F} E\right) \stackrel{1 \otimes N_{E / F}}{\longrightarrow} K_{2}(E)
$$

is surjective, which is obvious.

Proof of theorem 2. The statement is true for quadrics of dimension 0 by the long exact sequence for a quadratic extension [1, Satz 4.5] and [20]. If $\operatorname{dim} X=1$, then by [18, prop. 3.15] and the Milnor conjecture in degrees 2 and 3 ([15] and [20]), one has $\operatorname{Ker} \eta^{4}=c(q) \cdot H^{2} F$, where $q$ is a quadratic form defining $X$ and $c(q)$ is its Clifford invariant. Theorem 2 follows from this by reapplying [15]. If $\operatorname{dim} X=2$ and $X$ is defined by a 2-fold Pfister form, then $\operatorname{Ker} \eta_{X}^{4}=\operatorname{Ker} \eta_{C}^{4}$ where $C$ is a conic traced on $X$ [10, prop. $2.5 \mathrm{c})$ ], so theorem 2 follows from the one-dimensional case.

Finally, let $X$ be defined by $q=\langle d,-a,-b, a b\rangle$, with $d \notin F^{* 2}$, and let $E=F(\sqrt{d})$. Note that $q_{E}$ is the 2-fold Pfister form $\ll a, b \gg$, so $\operatorname{Ker}\left(H^{4} E \rightarrow H^{4} E(X)\right)=$ $(a, b) \cdot H^{2} E$ by the above. Let $(a, b) \cdot x \in \operatorname{Ker}\left(H^{4} E \rightarrow H^{4} E(X)\right)$, with $x \in H^{2} E$. By [15], $x$ is a sum of symbols and, by [2, cor. 5.3], it is even a sum of symbols of the form $(y, e)$, with $y \in E^{*}$ and $e \in F^{*}$. Now

$$
\operatorname{Cor}_{E / F}(a, b, y, e)=\left(a, b, N_{E / F} y, e\right) .
$$

This proves both a) and b), since

$$
\left(a, b, N_{E / F} y\right)=\operatorname{Cor}_{E / F}(a, b, y) \in \operatorname{Ker}\left(H^{3} F \rightarrow H^{3} F(X)\right) .
$$


2.7. Remark. In characteristic 0, one can give an alternative proof of theorem 2 by using the results of [9]. By loc. cit., cor. 5.5, there is (for any quadric $X$ ) an exact sequence after localisation at 2 :

$$
0 \rightarrow H^{1}\left(X, \mathcal{K}_{3}\right) \rightarrow K_{2}\left(E_{1}\right) \stackrel{\delta}{\rightarrow} \operatorname{Ker} \eta^{4} \rightarrow H^{2}\left(X, \mathcal{K}_{3}\right) \rightarrow H^{2}\left(\bar{X}, \mathcal{K}_{3}\right)
$$

where $E_{1}$ is a certain étale extension of $F$ associated to $X$. If $\operatorname{dim} X=2$, we have by [9, prop. 6.2, lemma 8.2 and cor. 8.6] (see also loc. cit., cor. 6.3)

$$
\begin{aligned}
E_{1}=E & =F(\sqrt{d}) \\
\delta(\{a, b\}) & =\operatorname{Cor}_{E / F}((a, b) \cdot c(q)) .
\end{aligned}
$$

On the other hand, the map $H^{2}\left(X, \mathcal{K}_{3}\right) \rightarrow H^{2}\left(\bar{X}, \mathcal{K}_{3}\right)$ is injective $(c f . \quad[20$, lemma $2.6])$.

\section{Coker $\eta^{4}$ : some preparations. Proof of theorem 3}

Let $X$ be a quadric over $F$. Recall that, by [9, th. 4.4], there is a spectral sequence for any $n \geq 0$

$$
E_{2}^{p, q}(X, n)=H_{\text {ét }}^{p-q}\left(F, C H^{q}\left(X_{s}\right) \otimes \mathbf{Z}(n-q)\right) \Rightarrow H^{p+q}
$$

with maps $H^{p+q} \rightarrow H_{\text {ét }}^{p+q}(X, \mathbf{Z}(n))$ which are bijective for $p+q \leq 2 n$ and injective for $p+q=2 n$. Here $H_{\text {ét }}^{*}(X, \mathbf{Z}(n))$ is étale motivic cohomology and $X_{s}=F_{s} \otimes_{F} X$, where $F_{s}$ is a separable closure of $F$. These spectral sequences are compatible with products and transfer.

Suppose $n \leq 3$. Then one has $H_{\text {ét }}^{n+1}(F, \mathbf{Z}(n)) \otimes \mathbf{Z}_{(2)}=0$ (Hilbert 90); from this one deduces that

$$
E_{2}^{n+q+1, q}(X, n+q) \otimes \mathbf{Z}_{(2)}=0
$$

for any $q \geq 0$ [9]. (In fact the restriction on $n$ is not necessary in view of Voevodsky's proof of the Milnor conjecture [25]; but we won't need this here.)

Specialising [9, cor. 5.5] to quadrics, dimension by dimension, and taking loc. cit., prop. 6.2, lemma 8.2 and cor. 8.6 into account, we obtain the complexes below after localisation at 2 .

\subsection{The cases $\operatorname{dim} X=2,3$; proof of theorem 3 .}

$\operatorname{dim} X=2$. Coker $\eta^{4}$ is a subgroup of the homology of

$$
H^{2}\left(X, \mathcal{K}_{3}\right) \stackrel{\xi^{4}}{\longrightarrow} F^{*} \stackrel{\delta}{\rightarrow} H^{3} E .
$$

Here $E=F(\sqrt{d}), \xi^{4}$ is the map $H^{2}\left(X, \mathcal{K}_{3}\right) \rightarrow H^{2}\left(\bar{X}, \mathcal{K}_{3}\right)^{G_{F}} \simeq F^{*}$ and $\delta(x)=$ $(x)_{E} \cdot c\left(q_{E}\right)$, where $q$ is a quadratic form defining $X ; \delta$ is the differential $d_{2}^{3,2}(X, 3)$ of the above spectral sequence. (Note that $C H^{1}(X) \rightarrow C H^{1}(\bar{X})$ is surjective [12] and $C H^{3}(X)=0$.) By [3, prop. 2.5], (6) is exact.

$\operatorname{dim} X=3$. Coker $\eta^{4}$ is a subgroup of the homology of

$$
H^{2}\left(X, \mathcal{K}_{3}\right) \stackrel{\xi^{4}}{\longrightarrow} F^{*} \stackrel{\delta}{\rightarrow} H^{3} F
$$


Notation is as above, except that $\delta(x)=(x) \cdot c(q)$. (Note that $C H^{1}(X) \rightarrow C H^{1}(\bar{X})$ is surjective and $C H^{3}(X)_{\text {tors }}=0$ [12].) By [10, computation before cor. 5.3], (7) is exact.

We have proven theorem 3 .

3.2. The case $\operatorname{dim} X=4$.

$\operatorname{dim} X=4$. There is a complex

$$
0 \rightarrow \text { Coker } \eta^{4} \rightarrow C H^{3}(X)_{\text {tors }} \rightarrow H^{6}(X, \mathbf{Z}(3))
$$

and the kernel of the first map is a subgroup of the homology of

$$
H^{2}\left(X, \mathcal{K}_{3}\right) \stackrel{\xi^{4}}{\rightarrow} E^{*} \stackrel{\delta}{\rightarrow} H^{3} F
$$

Notation is as above, except that $\delta(x)=\operatorname{Cor}_{E / F}\left((x) \cdot c\left(q_{E}\right)\right)$. (Note that $C H^{1}(X) \rightarrow$ $C H^{1}(\bar{X})$ is surjective.) By $[12$, cor. 4.5 , th. 7.3 and remark 7.2$], C H^{3}(X)_{\text {tors }}=\mathbf{Z} / 2$ if $X$ is intermediate or a neighbour, and 0 otherwise.

In the Albert and virtual Albert cases, we shall need the following more precise fact $(i b i d$.$) . The differential d_{3}^{3,2}(X, 3)$ of the spectral sequence (4) acts as

$$
\operatorname{Ker} \delta \stackrel{d_{3}^{3,2}(X, 3)}{\longrightarrow} H^{5}(F, 3)
$$

one has $\operatorname{Im} \xi^{4} \subseteq \operatorname{Ker} d_{3}^{3,2}(X, 3)$ and the kernel of the first map in (8) is isomorphic to $\operatorname{Ker} d_{3}^{3,2}(X, 3) / \operatorname{Im} \xi^{4}$. We shall see that, in fact, $d_{3}^{3,2}(X, 3)=0$ for any 4-dimensional quadric $X$.

We shall need the following lemma here only for $n=1,2(n=2$ for the proof of proposition 4.5, $n=1$ for the proof of lemma 4.17).

3.1. Lemma. Let $X$ be a 4-dimensional quadric and $h$ be a hyperplane section of $X$ and $\mathrm{cl}^{1}(h)$ its class in $H^{2}(X, \mathbf{Z}(1))$. Let $\pi: X \rightarrow$ SpecF be the structural map and $\beta: \mathbf{Z} / 2_{\text {ét }} \simeq \mathbf{Z} / 2(n)_{\text {ét }} \rightarrow \mathbf{Z}(n)[1]_{\text {ét }}$ the integral Bockstein. Then the sequence

$$
H^{n-1}(E, \mathbf{Z} / 2) \stackrel{A}{\rightarrow} H^{n+2}(F, \mathbf{Z}(n)) \stackrel{B}{\rightarrow} H^{n+4}(X, \mathbf{Z}(n+1))
$$

is exact for $0 \leq n \leq 3$, where $A(x)=\beta \operatorname{Cor}_{E / F}\left(x \cdot c\left(q_{E}\right)\right)$ and $B(y)=\left(\pi^{*} y\right) \cdot c l^{1}(h)$.

Proof. Let $F^{p} H^{m}(X, \mathbf{Z}(n))$ be the (decreasing) filtration induced on $H^{m}(X, \mathbf{Z}(n))$ by the spectral sequence (4). By multiplicativity, the image of $B$ is contained in $F^{n+2} H^{n+4}(X, \mathbf{Z}(n+1))$. The factor group

$$
F^{n+2} H^{n+4}(X, \mathbf{Z}(n+1)) / F^{n+3} H^{n+4}(X, \mathbf{Z}(n+1))=E_{\infty}^{n+2,2}(X, n+1)
$$

is a subquotient of $E_{2}^{n+2,2}(X, n+1)$. For $n \leq 4$, the latter is 0 by Hilbert 90 (see (5); remember that everything is localised at 2), hence $F^{n+2} H^{n+4}(X, \mathbf{Z}(n+1))=$ $F^{n+3} H^{n+4}(X, \mathbf{Z}(n+1))$. Dividing by $F^{n+4}$, we therefore get an induced map

$$
H^{n+2}(F, \mathbf{Z}(n)) \stackrel{\bar{B}}{\rightarrow} E_{\infty}^{n+3,1}(X, n+1) .
$$


For $n \leq 3$, the differential hitting the corresponding $E_{r}$-term is 0 for $r>2$, by dimension counting. So, in this range, there is a commutative diagram

$$
\begin{array}{cc} 
& E_{2}^{n+3,1}(X, n+1) / \operatorname{Im} d_{2}^{n+1,2} \\
\nearrow & \uparrow \\
H^{n+2}(F, \mathbf{Z}(n)) \stackrel{\bar{B}}{\longrightarrow} & E_{\infty}^{n+3,1}(X, n+1)
\end{array}
$$

where the vertical arrow is injective. We have an isomorphism

$$
E_{2}^{n+3,1}(X, n+1) \simeq H^{n+2}\left(F, C H^{1}\left(X_{s}\right) \otimes \mathbf{Z}(n)\right) \simeq H^{n+2}(F, \mathbf{Z}(n)) .
$$

This isomorphism identifies the oblique map in (11) with the natural projection. Indeed, by multiplicativity, it suffices to check this for $n=0$. In that case we have $d_{2}^{n+1,2}=0$, and we are left to prove that the composition

$$
H^{2}(F, \mathbf{Z}) \stackrel{B}{\rightarrow} H^{4}(X, \mathbf{Z}(1)) \stackrel{e}{\rightarrow} H^{2}\left(F, C H^{1}\left(X_{s}\right)\right)
$$

is the identity (identifying $C H^{1}\left(X_{s}\right)$ with $\mathbf{Z}$ via its generator $h$ ), where $e$ is the edge homomorphism of the weight 1 spectral sequence. This is clear, again by multiplicativity. Lemma 3.1 follows, using [9, cor. 8.6 and 6.3] for the values of the $d_{2}$ differentials.

\section{Proof OF THEOREM 4}

4.1. Generalities. Let $X$ be a 4-dimensional quadric defined by the 6 -dimensional quadratic form $q$. We shall constantly use the Clifford algebra $C(q)$ and even Clifford algebra $C_{0}(q)$ of $q$, for which we refer to [14, ch. V] or [13]. Let $d=d_{ \pm} q$ and $E=F[t] /\left(t^{2}-d\right)$, so that

$$
E= \begin{cases}F(\sqrt{d}) & \text { if } d \notin F^{* 2} \\ F \times F & \text { if } d \in F^{* 2}\end{cases}
$$

Recall that $C(q)$ is a central simple algebra of degree 8 over $F$ and that $C_{0}(q)$ is an Azumaya algebra of degree 4 over $E$; if $d=1$, then $C(q) \simeq M_{2}(A)$ and $C_{0}(q) \simeq A \times A$, where $A$ is central simple of degree 4 over $F$. In general, by scaling $q$ (e.g. ensuring $1 \in D(q)$ ), we may and shall assume that ind $C(q) \leq 4$.

4.1. Proposition. In (9), we have

a) $\operatorname{Ker} \delta=\left\{a \in E^{*} \mid N_{E / F}(a) \in F^{* 2} \operatorname{Nrd} C(q)^{*}\right\}$.

b) $\operatorname{Im} \xi^{4}=F^{*} \operatorname{Nrd}_{C_{0}(q) / E} C_{0}(q)^{*}$.

Proof. a) For $a \in E^{*}$, we have

$$
\delta(a)=\operatorname{Cor}_{E / F}\left((a) \cdot c\left(q_{E}\right)\right)=\left(N_{E / F}(a)\right) \cdot c(q) .
$$

Since ind $C(q) \leq 4$, the sequence

$$
F^{* 2} \operatorname{Nrd} C(q)^{*} \rightarrow F^{*} \stackrel{\cdot c(q)}{\longrightarrow} H^{3} F
$$

is exact by $[17$, cor. to th. 7$]$, hence the claim. 
b) By the same arguments as in op. cit., pp. 74-75, we have

$$
K_{1}(X)^{(2)}=F^{*} h^{2} \oplus F^{*} h^{3} \oplus K_{1}\left(C_{0}(q)\right)
$$

where $h$ is the class of a hyperplane section. Over the separable closure $F_{s}$, we get

$$
K_{1}\left(X_{s}\right)^{(2)}=F_{s}^{*} h^{2} \oplus F_{s}^{*} h^{3} \oplus F_{s}^{*} P_{1} \oplus F_{s}^{*} P_{2}
$$

where $P_{1}, P_{2}$ are the classes of two conjugate plane sections. Therefore

$$
\left(K_{1}\left(X_{s}\right)^{(2)}\right)^{G_{F}} \simeq F^{*} h^{2} \oplus F^{*} h^{3} \oplus E^{*}
$$

and, for this decomposition, the map $K_{1}(X)^{(2)} \rightarrow\left(K_{1}\left(X_{s}\right)^{(2)}\right)^{G_{F}}$ induced by extension of scalars has matrix $\left(\begin{array}{ccc}I d & 0 & 0 \\ 0 & I d & 0 \\ 0 & 0 & \text { Nrd }\end{array}\right)$ (remember that $C_{0}(q)$ is an Azumaya algebra over $E$ ). On the other hand, $H^{2}\left(X_{s}, \mathcal{K}_{3}\right)=F_{s}^{*} \otimes C H^{2}\left(X_{s}\right)=F_{s}^{*} P_{1} \oplus F_{s}^{*} P_{2}$, and with this decomposition, the projection $K_{1}\left(X_{s}\right)^{(2)} \rightarrow H^{2}\left(X_{s}, \mathcal{K}_{3}\right)$ has matrix $\left(\begin{array}{cccc}I d & 0 & \text { Id } & 0 \\ \text { Id } & 0 & 0 & \text { Id }\end{array}\right)$. Hence the map $F^{*} h^{2} \oplus F^{*} h^{3} \oplus E^{*} \simeq\left(K_{1}\left(X_{s}\right)^{(2)}\right)^{G_{F}} \rightarrow H^{2}\left(X_{s}, \mathcal{K}_{3}\right)^{G_{F}} \simeq E^{*}$ has matrix $(\Delta 0 I d)$, where $\Delta$ is the embedding $F^{*} \rightarrow E^{*}$. Finally, the composite map $\Phi$ has matrix

$$
\left(\begin{array}{lll}
\Delta & 0 & I d
\end{array}\right)\left(\begin{array}{ccc}
I d & 0 & 0 \\
0 & I d & 0 \\
0 & 0 & \mathrm{Nrd}
\end{array}\right)=\left(\begin{array}{lll}
\Delta & 0 & N r d
\end{array}\right)
$$

By [17, prop. 2], $K_{1}(X)^{(2)} \rightarrow H^{2}\left(X, \mathcal{K}_{3}\right)$ is surjective, hence $\Phi$ has the same image as $\xi^{4}$. The conclusion follows.

4.2. The neighbour case. It was proved in [10, th. $6(3)]$.

4.3. The intermediate case. The following lemma lifts a result of Szyjewski [23, prop. 3.3.6 b) and prop. 5.4.6] from mod 2 cohomology to motivic cohomology. For any smooth variety $X$ and $n \geq 0$, let

$$
c l^{n}: C H^{n}(X) \rightarrow H_{\text {ét }}^{2 n}(X, \mathbf{Z}(n))
$$

denote the étale motivic cycle map.

4.2. Lemma. Let $Y$ be the 3-dimensional quadric defined by any neighbour of the 3-fold Pfister form «a,b,c 》. Let $\pi: Y \rightarrow$ Spec $F$ be the structure map and $e$ be the non-zero torsion element of $\mathrm{CH}^{2}(Y)$ ([23, prop. 3.3.6 b)], [12, th. 6.1]). Then

$$
c l^{2}(e)=\pi^{*} \beta(a, b, c) .
$$

Here $(a, b, c) \in H^{3}(F, \mathbf{Z} / 2)=H^{3}(F, \mathbf{Z} / 2(2))$ is the cup-product of the Kummer classes of $a, b, c$ and $\beta: H^{3}(F, \mathbf{Z} / 2(2)) \rightarrow H^{4}(F, \mathbf{Z}(2))$ is the integral Bockstein.

Proof. The spectral sequence (4), together with [9, cor. 8.6], yields another exact sequence

$$
0 \rightarrow H^{4}(F, \mathbf{Z}(2)) \stackrel{\pi^{*}}{\longrightarrow} H^{4}(Y, \mathbf{Z}(2)) \rightarrow C H^{2}\left(Y_{F_{s}}\right)^{G_{F}}
$$


Let $K=F(Y)$. We get a commutative diagram with exact rows:

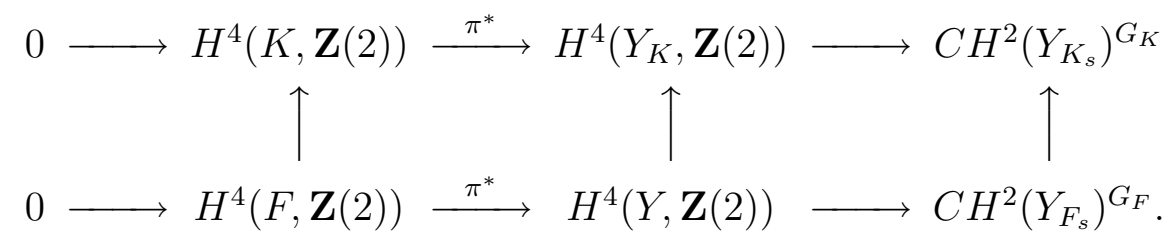

The right vertical arrow is an isomorphism. The left one coincides via the Bockstein map with

$$
H^{3}(F, \mathbf{Q} / \mathbf{Z}(2)) \rightarrow H^{3}(K, \mathbf{Q} / \mathbf{Z}(2)) .
$$

The kernel of this map is 2-torsion by the usual transfer argument. Since $H^{3}(F, \mathbf{Z} / 2)$ injects into $H^{3}(F, \mathbf{Q} / \mathbf{Z}(2))[15]$, it coincides with the kernel of

$$
H^{3}(F, \mathbf{Z} / 2) \rightarrow H^{3}(K, \mathbf{Z} / 2)
$$

which is generated by $(a, b, c)$ (Arason [1, Satz 5.6]). All this implies that

$$
\operatorname{Ker}\left(H^{4}(Y, \mathbf{Z}(2)) \rightarrow H^{4}\left(Y_{K}, \mathbf{Z}(2)\right)\right)=\left\langle\pi^{*} \beta(a, b, c)\right\rangle .
$$

Recall from [8] or [9] the short exact sequence

$$
0 \rightarrow C H^{2}(Y) \stackrel{c l^{2}}{\longrightarrow} H^{4}(Y, \mathbf{Z}(2)) \rightarrow H^{0}\left(Y, \mathcal{H}^{3}(\mathbf{Q} / \mathbf{Z}(2))\right) \rightarrow 0 .
$$

Since $Y_{K}$ is isotropic, $e_{K}=0$, hence $c l^{2}(e)=\pi^{*} \beta(a, b, c)$ since $c l^{2}$ is injective.

4.3. Remark. Lemma 4.2 shows incidentally that, if $\psi$ is any neighbour of a 3 -fold Pfister form $\varphi$, the map $C H^{2}\left(X_{\varphi}\right)_{\text {tors }} \rightarrow C H^{2}\left(X_{\psi}\right)_{\text {tors }}$ is an isomorphism.

4.4. Lemma. Let $q=\ll a, b \gg \perp\langle-c, c d\rangle$ be an anisotropic 6-dimensional quadratic form, and let $E=F(\sqrt{d})$. Then the equation

$$
(a, b, c)=\operatorname{Cor}_{E / F}\left(x \cdot(a, b)_{E}\right) \in H^{3}(F, \mathbf{Z} / 2)
$$

has no solution in $x \in H^{1}(E, \mathbf{Z} / 2)$.

Proof. Let $x$ be a solution; then

$$
(a, b, c N(x))=0 .
$$

By [20], this implies that the 3-fold Pfister form $\ll a, b, c N(x) \gg$ is hyperbolic, or that

$$
\ll a, b \gg \cong c N(x) \ll a, b \gg \text {. }
$$

Therefore

$$
\begin{aligned}
q & \cong c N(x) \ll a, b \gg \perp\langle-c, c d\rangle \cong c N(x)(\ll a, b \gg \perp N(x)\langle-1, d\rangle) \\
& \cong c N(x)(\ll a, b \gg \perp\langle-1, d\rangle)
\end{aligned}
$$

and the last form is isotropic, thereby contradicting the hypothesis.

4.5. Proposition. Let $X$ be intermediate. Then:

a) The map $C H^{3}(X)_{\text {tors }} \rightarrow H^{6}(X, \mathbf{Z}(3))$ of (8) is injective.

b) In (9), $\operatorname{Ker} \delta / \operatorname{Im} \xi^{4}=0$. 
Proof. a) Let $q^{\prime}$ be a 5-dimensional subform of a form $q$ defining $X$ which is a Pfister neighbour, and let $Y$ be the corresponding hyperplane section of $X$. Let $i$ be the corresponding closed immersion. We have a commutative diagram

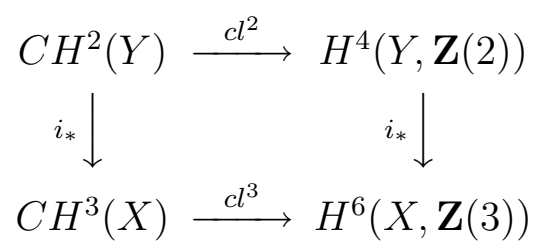

where both vertical maps are Gysin maps. Recall that $C H^{2}(Y)_{\text {tors }}$ and $C H^{3}(X)_{\text {tors }}$ are both isomorphic to $\mathbf{Z} / 2$ [12]. Let $e$ be the non-zero torsion element of $C H^{2}(Y)$. We shall show that $i_{*} c l^{2}(e) \neq 0$. This will both show that $i_{*}(e)$ is the generator $e^{\prime}$ of $C H^{3}(X)_{\text {tors }}$ and that $c l^{3}\left(e^{\prime}\right) \neq 0$.

By lemma $4.2, c l^{2}(e)=\pi_{Y}^{*} \beta(a, b, c)=i^{*} \pi_{X}^{*} \beta(a, b, c)$, where $\pi_{Y}$ (resp. $\left.\pi_{X}\right)$ is the structural map for $Y$ (resp. $X)$. Then, by the projection formula

$$
i_{*} c l^{2}(e)=\pi_{X}^{*} \beta(a, b, c) i_{*} i^{*}(1)=\pi_{X}^{*} \beta(a, b, c) c l^{1}(h) .
$$

By lemma 3.1 (applied for $n=2$ ), this element is 0 if and only if $\beta(a, b, c)=$ $\beta \operatorname{Cor}_{E / F}\left(x \cdot c\left(q_{E}\right)\right)$, with $x \in H^{1}(E, \mathbf{Z} / 2)$, that is, $(a, b, c)=\operatorname{Cor}_{E / F}(x \cdot(a, b))$. Since $X$ is anisotropic, this equation has no solution by lemma 4.4 .

b) By scaling $q$, we may assume that ind $C(q)=2$. Let $A$ be a quaternion algebra similar to $C(q)$. Let $a \in E^{*}$ be such that $N_{E / F}(a)=\operatorname{Nrd}(b)$, with $b \in A^{*}$. Choose a maximal subfield $L \subset A$ containing $b$, so that $\operatorname{Nrd}(b)=N_{L / F}(b)$. Let $K=E L$. By an easy consequence of [24, cor. 2.10], there is a pair $(\lambda, u) \in F^{*} \times K^{*}$ such that $a=\lambda N_{K / E}(u)$, hence $a=\lambda \operatorname{Nrd}(u)$, where $u$ is viewed as an element of $E \otimes_{F} A \sim C_{0}(q)$. We are therefore through by proposition $4.1 \mathrm{~b}$ )

Theorem 4 in the intermediate case follows from (8), (9) and proposition 4.5.

\subsection{The Albert case.}

4.6. Lemma. (Rost) Let $q$ be an Albert form. Then

$$
\operatorname{Sn}(q)=\left\{f \in F^{*} \mid f^{2} \in \operatorname{Nrd} C(q)^{*}\right\} .
$$

This lemma can be found in [13, prop. 16.6]; for the reader's convenience we give a self-contained proof. Let

$$
S \Gamma(q)=\left\{x \in C_{0}(q)^{*} \mid x V x^{-1}=V\right\}
$$

be the special spinor group of $q$, where $V \subset C_{1}(q)$ is the underlying vector space of $q$ (see $[10, \S 4]$ ). Let $x \mapsto x^{t}$ be the unique involution of $C(q)$ whose retriction to $V$ is the identity, and $\operatorname{sn}(x)=x x^{t}$. As is well-known, $\operatorname{sn}(x) \in F^{*}$ for $x \in S \Gamma(q)$ and

$$
\operatorname{Sn}(q)=\operatorname{sn}(S \Gamma(q)) \text {. }
$$

We have

$$
C_{0}(q) \simeq A \times A, \quad C(q) \simeq M_{2}(A)
$$


for a biquaternion algebra $A$; in particular, $\operatorname{Nrd} C(q)^{*}=\operatorname{Nrd} A^{*}$. By [10, cor. 4.3], there is an exact sequence

$$
1 \rightarrow S \Gamma(q) \stackrel{\left(p_{1}, s n\right)}{\longrightarrow} A^{*} \times F^{*} \stackrel{\omega}{\longrightarrow} F^{*}
$$

where $p_{1}$ is given by the first projection $C_{0}(q) \rightarrow A$ and $\omega(a, f)=\operatorname{Nrd}(a) / f^{2}$. Lemma 4.6 follows tautologically from this exact sequence.

4.7. Lemma. Let $X$ be a non hyperbolic Albert quadric. Then $d_{3}^{2,2}(X, 2)=0$ in the motivic spectral sequence (4).

Of course, the lemma also holds if $X$ is hyperbolic, but we won't have to use this fact.

Proof. Recall the complex

$$
C H^{2}(X) \stackrel{\xi^{3}}{\longrightarrow} C H^{2}\left(X_{s}\right)^{G_{F}} \stackrel{d_{2}^{2,2}(X, 2)}{\longrightarrow} \operatorname{Br}(F)
$$

from $[9,5.3]$. By loc.cit., there is an exact sequence

$$
0 \rightarrow \text { Coker } \eta^{3} \rightarrow H \stackrel{d_{3}^{2,2}}{\longrightarrow} H^{4}(F, 2) .
$$

with $H:=\operatorname{Ker} d_{2}^{2,2} / \operatorname{Im} \xi^{3}$.

By a computation similar to that of proposition 4.1, we have

$$
\begin{array}{rlr}
\operatorname{Ker} d_{2}^{2,2}(X, 2) & =\left\{a P_{1}+b P_{2} \mid a \equiv b\right. & (\bmod 2)\} \\
\operatorname{Im} \xi^{3} & =\left\{a P_{1}+b P_{2} \mid a \equiv b\right. & (\bmod m)\} .
\end{array}
$$

as subgroups of $C H^{2}\left(X_{s}\right)^{G_{F}}=\mathbf{Z} P_{1} \oplus \mathbf{Z} P_{2}$. Here $m= \begin{cases}4 & \text { if } X \text { is anisotropic } \\ 2 & \text { if } X \text { is isotropic. }\end{cases}$ Indeed, the first equality follows trivially from $d_{2}^{2,2}\left(P_{1}\right)=d_{2}^{2,2}\left(P_{2}\right)=c(X) \neq 0[9$, th. 8.3]; the other one follows from $C H^{2}(X)=\left\langle h^{2}, m P_{1}\right\rangle[12]$ (recall that $h^{2}=P_{1}+P_{2}$ ). This shows that

$$
H \simeq \begin{cases}\mathbf{Z} / 2 & \text { if } X \text { is anisotropic } \\ 0 & \text { if } X \text { is isotropic. }\end{cases}
$$

On the other hand, if $X$ is anisotropic then Coker $\eta^{3} \simeq \mathbf{Z} / 2$ by [10, th. 5], and if $X$ is isotropic then Coker $\eta^{3}=0$. This shows that the first map in (12) is bijective, hence the claim.

4.8. Proposition. Let $X$ be an anisotropic Albert quadric. Then:

a) $d_{3}^{3,2}(X, 3)=0$ in $(10)$.

b) $C H^{3}(X)_{\text {tors }}=0$.

Proof. a) We have a commutative diagram of exact sequences (defining $H^{\prime}$ )

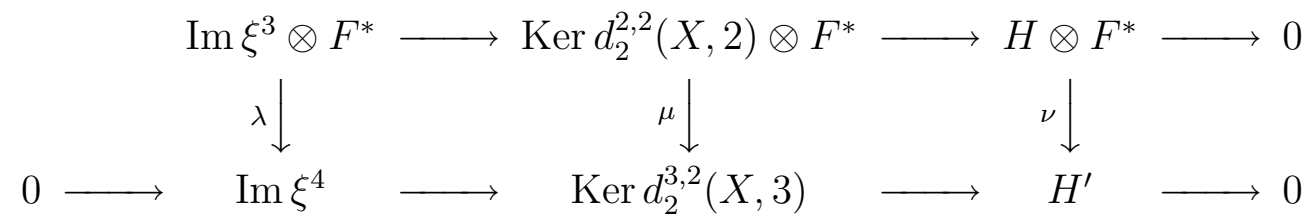


where the vertical arrows are given by cup-product. This first yields an exact sequence

$$
\text { Coker } \lambda \rightarrow \text { Coker } \mu \rightarrow \text { Coker } \nu \rightarrow 0 \text {. }
$$

Proposition 4.1 and the computation in the proof of lemma 4.7 identify the left square of (13) with

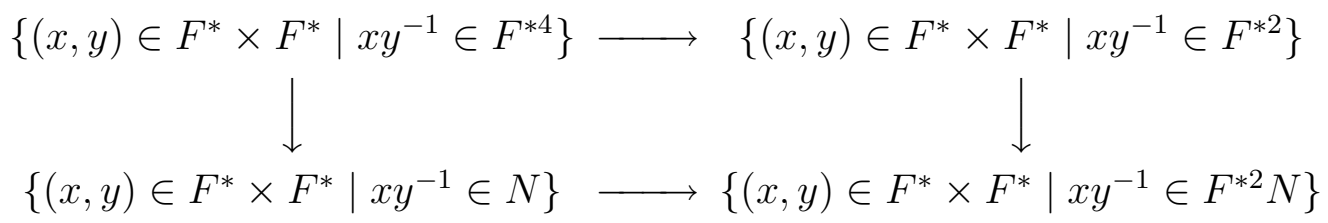

where $N:=\operatorname{Nrd} C(q)^{*}$, hence the first map of (14) with

$$
N / F^{* 4} \rightarrow F^{* 2} N / F^{* 2} \text {. }
$$

This shows that $\nu$ is surjective. By lemma 4.7 and the multiplicativity of the spectral sequences, this proves a).

b) This follows from Karpenko [12].

4.9. Remark. For future reference, we give a precise description of the map $\nu$ in diagram (13). By the computation in the proof of lemma 4.7, the generator $e$ of $H$ is represented by $2 P_{2} \in \operatorname{Ker} d_{2}^{2,2}(X, 2)$. Therefore, for $f \in F^{*}, \nu(e \otimes f)$ is represented by $\left(1, f^{2}\right)$ in $\operatorname{Ker} d_{2}^{3,2}(X, 3) \subseteq F^{*} \times F^{*}, c f$. (9).

Proof of theorem 4 in the case of an Albert quadric. In view of proposition 4.8 and (8), (9), (10), the group $H^{\prime}$ in (13) can be identified with Coker $\eta^{4}$. In the course of the proof of proposition 4.8 a), we have seen that the map $\nu$ in (13) is surjective; hence we are left to prove that $\operatorname{Ker} \nu=S n(q)$. This is a consequence of the following diagram chase. We have $H \otimes F^{*} \sim F^{*} / F^{* 2}$, induced by cup-product

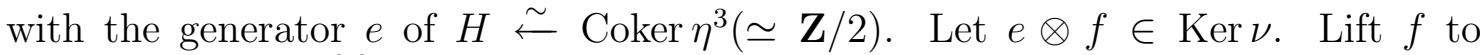
$\left(2 P_{2}\right) \otimes f \in \operatorname{Ker}_{2}^{2,2}(X, 2) \otimes F^{*}\left(\right.$ see remark 4.9). Then $\mu\left(\left(2 P_{2}\right) \otimes f\right)=\left(1, f^{2}\right) \in$ $\operatorname{Ker} d_{2}^{3,2}(X, 3)$, viewed as a subgroup of $F^{*} \times F^{*}$ (compare proposition $\left.4.1 \mathrm{a}\right)$ ). By assumption, $\left(1, f^{2}\right) \in \operatorname{Im} \xi^{4}$, which means that $f^{2} \in N$. But this condition is equivalent to $f \in S n(q)$ by lemma 4.6.

\subsection{The virtual Albert case.}

In this subsection, we let

$$
H^{\prime}:=\operatorname{Ker} d_{2}^{3,2}(X, 3) / \operatorname{Im} \xi^{4}
$$

denote the homology of the exact sequence (9). Recall, for a quadratic form $q$, the group of similarities of $q$

$$
G(q)=\left\{a \in F^{*} \mid a q \simeq q\right\} .
$$

4.10. Lemma. Let $q$ be an even dimensional form of discriminant $d \neq 1$, and let $E=$ $F(\sqrt{d})$. Then $G(q) \subseteq N_{E / F}\left(E^{*}\right)$.

Proof. Let $a \in G(q)$. Then $\langle 1,-a\rangle \otimes q \sim 0$. The lemma follows from taking the Clifford invariant of both sides (the left one is $(a, d)$ ). 
4.11. Lemma. Let $X$ be virtual Albert, $d$ its discriminant and $E=F(\sqrt{d})$. Then, in (9),

$$
\operatorname{Ker} \delta=N_{E / F}^{-1}(G(q)) \text {. }
$$

Proof. By the description of $\delta=d_{2}^{3,2}(X, 3)$ (see section 3),

$$
\operatorname{Ker} \delta=\left\{a \in E^{*} \mid N_{E / F}(a) \cdot c(q)=0\right\} .
$$

Let $a \in \operatorname{Ker} \delta$ and

$$
\varphi=\langle 1,-N(a)\rangle \otimes q .
$$

Then $\varphi$ is a 12-dimensional form in $I^{2} F$. We have

$$
e^{2}(\varphi)=e^{1}(\langle 1,-N(a)\rangle) \cdot e^{1}(q)=(N(a)) \cdot(d)=0,
$$

so $\varphi \in I^{3} F$. We also have $\ll N(a), d \gg \sim 0$, hence $\varphi \sim\langle 1,-N(a)\rangle \otimes(q \perp\langle 1,-d\rangle)$. Now

$$
\begin{aligned}
e^{3}(\varphi)=e^{3}(\langle 1,-N(a)\rangle \otimes & (q \perp\langle 1,-d\rangle)) \\
& =e^{1}(\langle 1,-N(a)\rangle) \cdot e^{2}(q \perp\langle 1,-d\rangle)=(N(a)) \cdot c(q)=0 .
\end{aligned}
$$

Hence $\varphi \in I^{4} F$ and, by the Arason-Pfister Hauptsatz, $\varphi \sim 0$. Therefore

$$
q \cong N(a) q
$$

which shows that $\operatorname{Ker} \delta \subseteq N^{-1}(G(q))$. The opposite inclusion is proved by reversing this argument.

4.12. Lemma. Let $E^{1}=\left\{x \in E^{*} \mid N_{E / F}(x)=1\right\}$. Then there is an exact sequence

$$
1 \rightarrow E^{1} \cap\left(F^{*} \operatorname{Nrd}_{E} C_{0}(q)^{*}\right) \rightarrow E^{1} \stackrel{\theta}{\rightarrow} H^{\prime} \rightarrow P S O(q, F) / R \rightarrow 0
$$

where $R$ denotes $R$-equivalence.

Proof. By [19, p. 203, cor. to lemma 5 and bottom of same page], we have

$$
P S O(q, F) / R \simeq P \operatorname{Sim}_{+}(q)(F) / R \simeq G(q) / F^{* 2} N_{E / F}\left(\operatorname{Nrd} C_{0}(q)^{*}\right) .
$$

By lemma 4.11 and proposition $4.1, H^{\prime} \simeq N^{-1}(G(q)) / F^{*} \operatorname{Nrd}_{E} C_{0}(q)$. Therefore the norm induces a map from $H^{\prime}$ to $\operatorname{PSim}_{+}(q)(F) / R$ : this is the map at the right of the exact sequence. This map is surjective by lemma 4.10. The exactness at the other terms is obvious.

We now want to prove that $d_{3}^{3,2}(X, 3)=0$ for a virtual Albert quadric $X$. We shall deduce this from the vanishing of $d_{3}^{3,2}(U, 2)$ for the affine quadric

$$
U=X \backslash Z
$$

where $Z$ is a nonsingular hyperplane section of $X$ and $d_{3}^{3,2}(U, 2)$ is a differential in an analogue of (4) for $U$. The use of $U$ was suggested by Rost. Although $U$ is not a geometrically cellular variety in the sense of [9], we shall see that the analogues of the spectral sequences of loc. cit. for $U$ are particularly simple. Let

$$
\tilde{\mathbf{Z}}=f_{*} \mathbf{Z} / \mathbf{Z}
$$

where $f$ is the projection Spec $E \rightarrow \operatorname{Spec} F$. As a $G_{F}$-module, $\tilde{\mathbf{Z}}$ is a free abelian group of rank 1 ; if $d \neq 1$, the Galois action is given by $g x=\varepsilon(g) x$, where $\varepsilon: G_{F} \rightarrow$ 
$\mathbf{Z} / 2$ is the character corresponding to the quadratic extension $E / F$. Otherwise, the Galois action is trivial. We have

4.13. Lemma. For all $p \geq 0$, the map

$$
C H^{p-1}\left(Z_{s}\right) \stackrel{i_{*}}{\rightarrow} C H^{p}\left(X_{s}\right)
$$

is injective. Its cokernel $C H^{p}\left(U_{s}\right)$ is

- $\mathbf{Z}$ for $p=0$

- $\tilde{\mathbf{Z}}$ for $p=2$

- 0 otherwise.

Proof. The claim is obvious for $p=0$. Suppose $p>0$. Let $h$ be the class of a hyperplane section of $X$ in $C H^{1}\left(X_{s}\right)$. By the projection formula, the map $i_{*}$ sends $h^{i}$ to $h^{i+1}$ for all $i \geq 0$. We now go case by case:

- $p=1$. The claim is clear, since $C H^{1}\left(X_{s}\right)$ has basis $h$.

- $p=2$. The group $C H^{2}\left(X_{s}\right)$ has basis $\left(P_{1}, P_{2}\right)$ with $P_{1}+P_{2}=h^{2}$. The claim follows.

- $p=3,4$. The claim is clear, since $C H^{p-1}\left(Z_{s}\right)$ (resp. $\left.C H^{p}\left(X_{s}\right)\right)$ is generated by $\frac{1}{2} h^{p-1}\left(\right.$ resp. $\left.\frac{1}{2} h^{p}\right)$.

4.14. Proposition. For all $n \geq 0$, there is a spectral sequence

$$
E_{2}^{p, q}(U, n) \Rightarrow H^{p+q}
$$

with maps $H^{p+q} \rightarrow H_{\text {ét }}^{p+q}(U, \mathbf{Z}(n))$ which are bijective for $p+q \leq 2 n$. The $E_{2}$-terms are as follows:

$$
E_{2}^{p, q}(U, n)= \begin{cases}H_{\text {ét }}^{p}(F, \mathbf{Z}(n)) & \text { for } q=0 \\ H_{\text {ét }}^{p-2}(F, \tilde{\mathbf{Z}}(n-2)) & \text { for } q=2 \\ 0 & \text { otherwise }\end{cases}
$$

For the proof, see Appendix A.

4.15. Remark. After the fact, we see that the $E_{2}$-terms can also be written as

$$
E_{2}^{p, q}(U, n)=H_{\text {ét }}^{p-q}\left(F, C H^{q}\left(U_{s}\right) \otimes \mathbf{Z}(n-q)\right)
$$

just as in [9] for a geometrically cellular variety. This formula is perhaps more mnemotechnical; however, the reader should be aware that it is a sort of 'miracle' (it would definitely not hold if the Chow groups of $U_{s}$ had torsion). 
Take $n=2,3$. Let us reproduce for $U$ part of the diagrams corresponding to those in $[9,5.3$ and 5.4] (here, all groups are localised at 2):

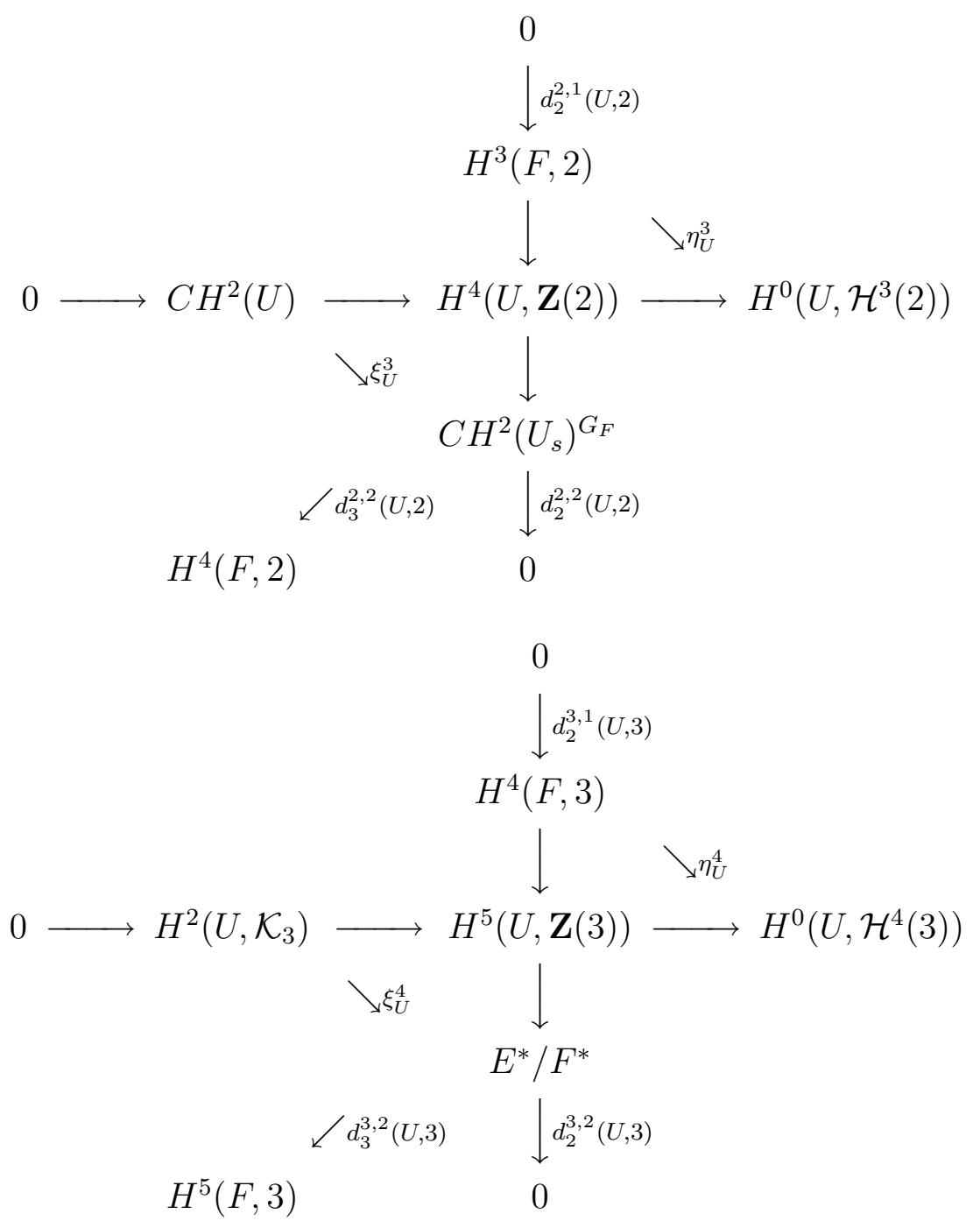

If $d=1$, the action of $G_{F}$ on $C H^{2}\left(X_{s}\right), C H^{2}\left(U_{s}\right)$ and $C H^{1}\left(Z_{s}\right)$ is trivial.

4.16. Lemma. Suppose $X$ is a non hyperbolic Albert quadric. Then

a) The composition

$$
\operatorname{Ker} d_{2}^{2,2}(X, 2) \subset C H^{2}\left(X_{s}\right) \rightarrow C H^{2}\left(U_{s}\right)
$$

has cokernel $\mathbf{Z} / 2$.

b) $\operatorname{Coker} \xi_{U}^{3} \neq 0$.

Proof. a) By lemma 4.13, the map $C H^{2}\left(X_{s}\right) \rightarrow C H^{2}\left(U_{s}\right)$ sends both basis elements $P_{1}, P_{2}$ to the same generator of $C H^{2}\left(U_{s}\right)$. The claim then follows from the computation of $\operatorname{Ker} d_{2}^{2,2}(X, 2)$ in the proof of lemma 4.7 (note that this computation only uses the fact that $c(q) \neq 0$ for a quadratic form $q$ defining $X)$. 
b) Consider the commutative diagram with exact rows

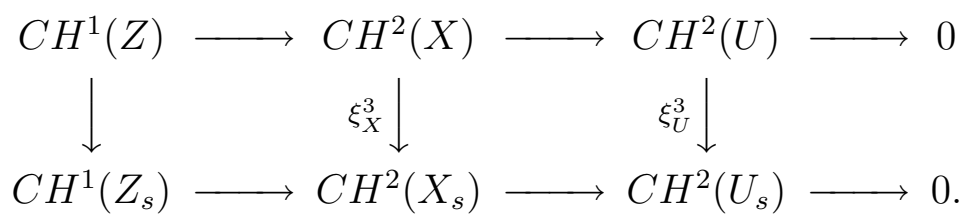

By [12] (compare [10, prop. $1.1 \mathrm{~b})]$ ), the left vertical map is surjective, hence Coker $\xi_{X}^{3} \stackrel{\sim}{\rightarrow}$ Coker $\xi_{U}^{3}$. The former is isomorphic to $\mathbf{Z} / 4$ or $\mathbf{Z} / 2$ according as $X$ is anisotropic or isotropic (ibid.).

4.17. Lemma. Suppose $X$ is a non hyperbolic Albert quadric. Then the cokernel of Coker $\eta_{X}^{3} \rightarrow$ Coker $\eta_{U}^{3}$ contains a nonzero element of order 2 .

Proof. By the "purity" exact sequence in $\mathcal{H}$-cohomology, we have a diagram

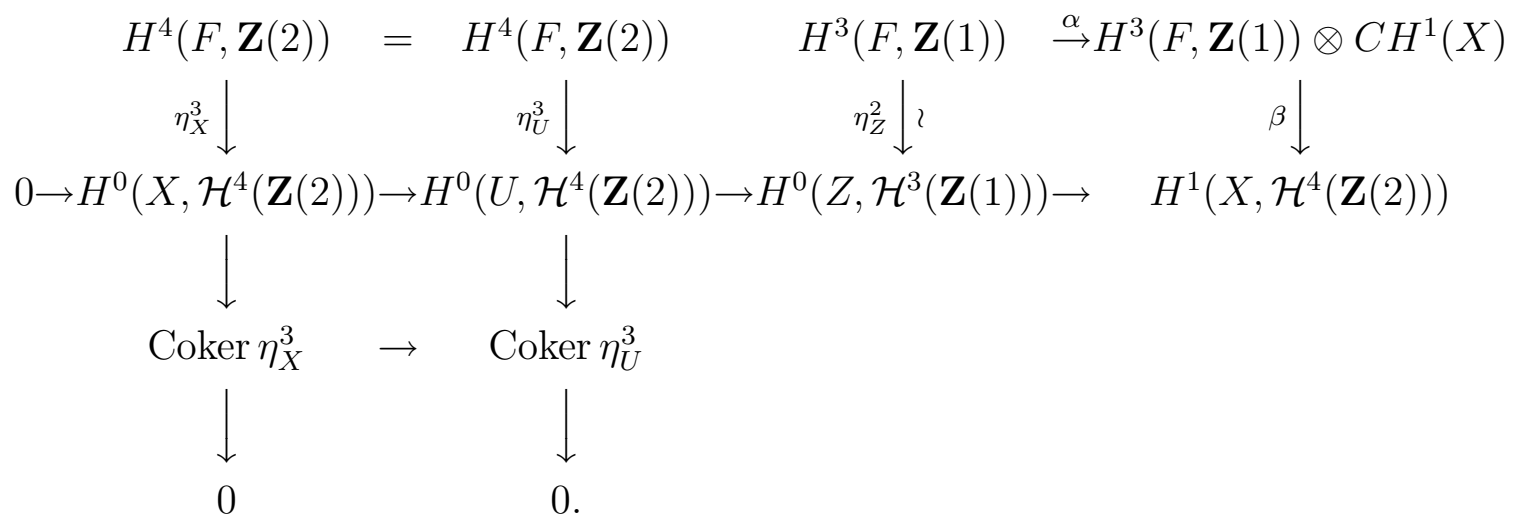

In this diagram, the middle row is exact; the map $\eta_{Z}^{2}$ is bijective by [1, comments after Satz 4.1] and [10, th. 4]. The map $\alpha$ (resp. $\beta$ ) is defined by tensoring with $h$ (resp. by $\beta(x \otimes y)=\pi^{*} x \cdot y$, where $\pi$ is the structural map of $X$ ). One checks easily that the square involving $\eta_{Z}^{2}, \alpha$ and $\beta$ commutes.

The diagram then yields an exact sequence

$$
0 \rightarrow \text { Coker } \eta_{X}^{3} \rightarrow \text { Coker } \eta_{U}^{3} \rightarrow \operatorname{Ker}(\beta \alpha) \rightarrow 0 .
$$

Consider the map $B: H^{3}(F, \mathbf{Z}(1)) \rightarrow H^{5}(X, \mathbf{Z}(2))$ of lemma 3.1. Letting $G^{p} H^{5}(X, \mathbf{Z}(2))$ denote the (decreasing) filtration defined on $H^{5}(X, \mathbf{Z}(2))$ by the coniveau spectral sequence, we have $\operatorname{Im} B \subseteq G^{1} H^{5}(X, \mathbf{Z}(2))$. By dimension counting, $E_{\infty}^{1,4} \hookrightarrow E_{2}^{1,4} \simeq$ $H^{1}\left(X, \mathcal{H}^{4}(\mathbf{Z}(2))\right)$ and, again by multiplicativity, the diagram

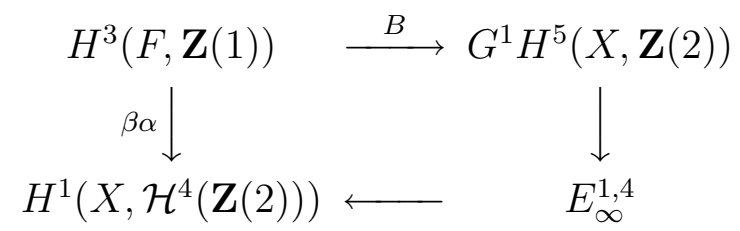

commutes. In particular, $\operatorname{Ker} B \subseteq \operatorname{Ker} \beta \alpha$. By lemma 3.1, $\operatorname{Ker} B=\{0, c(q)\}$. Lemma 4.17 follows.

4.18. Lemma. Suppose $X$ is a non-hyperbolic Albert quadric. Then $d_{3}^{2,2}(U, 2)=0$. 
Proof. Consider the commutative diagram with exact rows

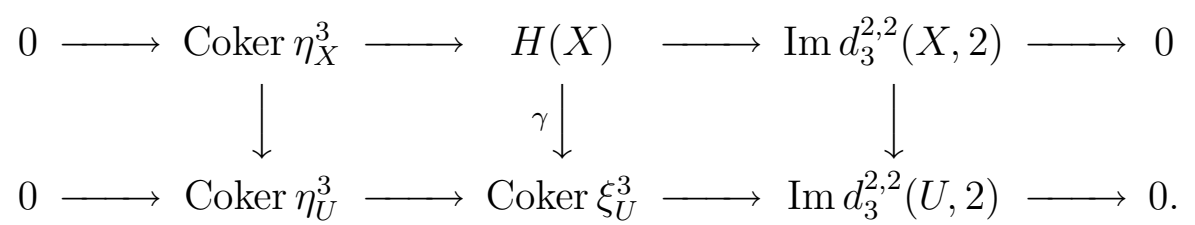

Here $H(X)=\operatorname{Ker} d_{2}^{2,2}(X, 2) / \operatorname{Im}\left(\xi_{X}^{3}\right)$; we have $d_{2}^{2,2}(U, 2)=0$ because its target group is 0 . By lemma 4.7 we have $\operatorname{Im} d_{3}^{2,2}(X, 2)=0$. Therefore the snake lemma yields an exact sequence

$$
\text { Coker } \eta_{X}^{3} \rightarrow \text { Coker } \eta_{U}^{3} \rightarrow \text { Coker } \gamma \rightarrow \operatorname{Im} d_{3}^{2,2}(U, 2) \rightarrow 0 .
$$

But lemma 4.16 shows that Coker $\gamma$ is isomorphic to $\mathbf{Z} / 2$. Lemma 4.17 now shows that $\operatorname{Im} d_{3}^{2,2}(U, 2)=0$, as desired (this proof also shows that the cokernel of Coker $\eta_{X}^{3} \rightarrow$ Coker $\eta_{U}^{3}$ is exactly of order 2 ).

4.19. Proposition. Let $X$ be virtual Albert. Then:

a) $C H^{3}(X)_{\text {tors }}=0$.

b) $d_{3}^{3,2}(X, 3)=0$.

Proof. a) This follows from Karpenko [12].

b) Diagram (16), the analogous diagram for $X[9,(5.4)]$ and functoriality give a commutative diagram

$$
\begin{array}{cc}
\operatorname{Ker} d_{2}^{3,2}(X, 3) & \longrightarrow E^{*} / F^{*} \\
d_{3}^{3,2}(X, 3) \downarrow & d_{3}^{3,2}(U, 3) \downarrow \\
H^{5}(F, 3) & =H^{5}(F, 3)
\end{array}
$$

which shows that it is sufficient to prove the vanishing of $d_{3}^{3,2}(U, 3)$, where $U$ is the complement of a hyperplane section as above. By a multiplicativity and transfer argument just as in [9, lemma 6.1], to do this it is enough to show that

$$
d_{3}^{2,2}\left(U_{K}, 2\right): C H^{2}\left(U_{s}\right)^{G_{K}} \rightarrow H^{4}(K, 2)
$$

is 0 for any finite extension $K / F$. If $E \nsubseteq K$, then $C H^{2}\left(U_{s}\right)^{G_{K}}=0$ and this is trivial. If $X_{K}$ is hyperbolic, then the computation of lemma 4.13 shows that $C H^{2}\left(U_{K}\right) \rightarrow C H^{2}\left(U_{s}\right)^{G_{K}}$ is bijective, hence the result again (cf. (15)). Finally, the remaining case is covered by lemma 4.18 .

4.20. Corollary. The map $H^{i}(F, \mathbf{Z}(3)) \rightarrow H^{i}(X, \mathbf{Z}(3))$ is injective for $i=5,6$.

Proof. For $i=5$, this is not a corollary of proposition 4.19: it follows from [9, cor. 8.6], which shows that the differential $d_{2}^{3,1}(X, 3)$ is 0 . For $i=6$, it follows from proposition 4.19 . 
For the next propositions, we have to describe precisely the restriction and corestriction

$$
\begin{aligned}
& \operatorname{Res}_{E / F}: H^{1}\left(F, C H^{2}\left(X_{s}\right) \otimes \mathbf{Z}(1)\right) \rightarrow H^{1}\left(E, C H^{2}\left(X_{s}\right) \otimes \mathbf{Z}(1)\right) \\
& \operatorname{Cor}_{E / F}: H^{1}\left(E, C H^{2}\left(X_{s}\right) \otimes \mathbf{Z}(1)\right) \rightarrow H^{1}\left(F, C H^{2}\left(X_{s}\right) \otimes \mathbf{Z}(1)\right)
\end{aligned}
$$

under the identification of these groups used in proposition 4.1; this is the most confusing part of this paper. We recall these identifications from [9]. We have

$$
H^{1}\left(F, C H^{2}\left(X_{s}\right) \otimes \mathbf{Z}(1)\right)=H^{0}\left(F, C H^{2}\left(X_{s}\right) \otimes F_{s}^{*}\right) .
$$

Taking the basis $\left(P_{1}, P_{2}\right)$ of $C H^{2}\left(X_{s}\right)$ consisting of the classes of two plane sections conjugate under $G_{F}$, the coefficient group gets identified with $\left(\mathbf{Z} P_{1} \oplus \mathbf{Z} P_{2}\right) \otimes F_{s}^{*}$. Since $G_{F}$ permutes $P_{1}$ and $P_{2}$, Galois invariants get identified with $E^{*}$ via the map

$$
x \mapsto P_{1} \otimes\{x\}+P_{2} \otimes\{\bar{x}\} .
$$

(We use the $K$-theoretic notation \{\} in order to avoid conflict between additive and multiplicative notation).

On the other hand, the identification of $H^{1}\left(E, C H^{2}\left(X_{s}\right) \otimes \mathbf{Z}(1)\right)=H^{0}\left(E, C H^{2}\left(X_{s}\right) \otimes\right.$ $\left.F_{s}^{*}\right)$ with $E^{*} \times E^{*}$ used in subsection 4.4 is given by the map

$$
(x, y) \mapsto P_{1} \otimes\{x\}+P_{2} \otimes\{y\} .
$$

The following lemma is now clear.

4.21. Lemma. Under the identifications above,

a) The action of $\operatorname{Gal}(E / F)$ on $E^{*} \times E^{*}$ is given by $(x, y) \mapsto(\bar{y}, \bar{x})$.

b) The restriction is given by the map

$$
\begin{aligned}
r: E^{*} & \rightarrow E^{*} \times E^{*} \\
x & \mapsto(x, \bar{x}) .
\end{aligned}
$$

c) The corestriction is given by the map

$$
\begin{aligned}
c: E^{*} \times E^{*} & \rightarrow E^{*} \\
(x, y) & \mapsto x \bar{y} .
\end{aligned}
$$

4.22. Proposition. The diagram

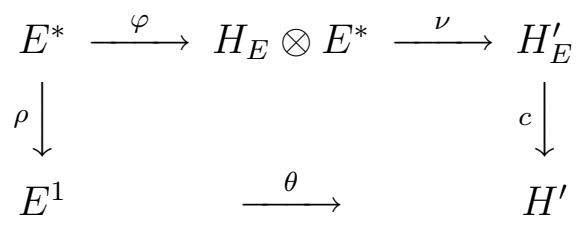

commutes, where $E^{1}=\operatorname{Ker} N_{E / F}$ as before, $H^{\prime}=\operatorname{Ker} \delta / \operatorname{Im} \xi^{4}, H_{E}^{\prime}$ is the corresponding group over $E, H_{E}=\operatorname{Ker} d_{2}^{2,2}\left(X_{E}, 2\right) / \operatorname{Im} \xi_{X_{E}}^{3}, \theta$ is the map of lemma 4.12, $\varphi(x)=e \otimes x$ (e is the generator of $H_{E}$ ), $\nu$ is the map of diagram (13), $\rho$ is the map $x \mapsto \bar{x} / x(\bar{x}$ is the conjugate of $x$ under $\operatorname{Gal}(E / F))$ and $c$ is induced by the map in lemma $4.21 \mathrm{c})$. 
Proof. By remark 4.9, we have $\nu \varphi(x)=\left(1, x^{2}\right)$ for $x \in E^{*}$, hence

$$
c(\nu \varphi(x)) \equiv \bar{x}^{2} \quad\left(\bmod \operatorname{Im} \xi^{4}\right) .
$$

On the other hand, $\theta \rho(x)$ is represented by $\bar{x} / x$ in $\operatorname{Ker} d_{2}^{3,2}(X, 2)$. But

$$
\bar{x} / x \equiv \bar{x}^{2} \quad\left(\bmod \operatorname{Im} \xi^{4}\right)
$$

since

$$
x \bar{x} \in F^{*} \subseteq \operatorname{Im} \xi^{4}
$$

by proposition 4.1 .

4.23. Proposition. Let $x \in \operatorname{Ker} \delta$. Then, with notation as in lemma 4.21 b) and proposition 4.22,

$$
r(x) \equiv \nu \varphi(x y) \quad\left(\bmod \operatorname{Im} \xi_{E}^{4}\right)
$$

where $y \in F^{*}$ is such that $N_{E / F}(x) y^{2}=N_{E / F}(x y) \in \operatorname{Nrd} C(q)^{*}$ (compare proposition 4.1).

Proof. We have

$$
r(x)=(x, \bar{x})
$$

and

$$
\nu \varphi(x y)=\left(1, x^{2} y^{2}\right)
$$

We claim that $(x, \bar{x}) \equiv\left(1, x^{2} y^{2}\right)\left(\bmod \operatorname{Im} \xi_{E}^{4}\right)$, or $\left(x, \bar{x} / x^{2} y^{2}\right) \in \operatorname{Im} \xi_{E}^{4}$. By proposition 4.1 , this is equivalent to

$$
\frac{x}{\bar{x} / x^{2} y^{2}}=x^{3} y^{2} / \bar{x}=(x y)^{4} / N_{E / F}(x) y^{2} \in \operatorname{Nrd} C\left(q_{E}\right)^{*} .
$$

Clearly, $(x y)^{4} \in \operatorname{Nrd} C\left(q_{E}\right)^{*}$, and $N_{E / F}(x) y^{2} \in \operatorname{Nrd} C(q)^{*} \subseteq \operatorname{Nrd} C\left(q_{E}\right)^{*}$.

Proof of theorem 4 in the case of a virtual Albert quadric. By proposition 4.19 and (8), (9), (10), Coker $\eta^{4}$ is identified with $H^{\prime}=\operatorname{Ker} \delta / \operatorname{Im} \xi^{4}$ in (9), just as Coker $\eta_{X_{E}}^{4}$ is identified with $H_{E}^{\prime}$ by the Albert quadric case. Putting lemma 4.12 and proposition 4.19 together, we get a commutative diagram with exact rows

$$
\begin{array}{ll}
E^{*} \stackrel{\nu \varphi}{\longrightarrow} \text { Coker } \eta_{E}^{4} \\
\rho \downarrow & \operatorname{Cor}_{E / F} \downarrow \\
E^{1} \stackrel{\theta}{\longrightarrow} \text { Coker } \eta^{4} \longrightarrow \operatorname{PSO}(q, F) / R \rightarrow 0 .
\end{array}
$$

The exactness of the sequence of theorem 4 follows from (18) and Hilbert's theorem 90 (classical style), which asserts that $\rho$ is surjective.

\section{Proofs of theorems 1 And 5}

5.1. Proof of theorem 1. This should have been already noticed in [10]. Just notice that, in the diagram of [10, top of p. 867], the zero can be removed from $H^{i}\left(F, \mu_{m}^{\otimes(i-1)}\right)_{0}$ because, under the assumption, the Bockstein that follows is 0 (compare loc. cit., proof of prop. 7.3). We get the claim by passing to the limit over $m$ in this diagram. 


\subsection{Proof of theorem 5.}

5.1. Lemma. Let $X$ be a smooth variety such that $C H^{1} X \rightarrow C H^{1} X_{s}$ and $C H^{2} X / 2 \rightarrow$ $\left(C H^{2} X_{s} / 2\right)^{G_{F}}$ are surjective. Then there is an exact sequence

$$
H^{4} F \stackrel{\eta_{2}^{4}}{\longrightarrow} H^{0}\left(X, \mathcal{H}^{4}\right) \stackrel{d_{2}^{2,3}}{\longrightarrow} H^{2}\left(X, \mathcal{H}^{3}\right) \stackrel{\varphi}{\rightarrow} H^{5} X
$$

where $d_{2}^{2,3}$ is a differential in the coniveau spectral sequence for $\mathbf{Z} / 2$ coefficients and $\varphi$ is an edge homomorphism in the same spectral sequence. Therefore,

$$
\text { Coker } \eta_{2}^{4} \stackrel{\sim}{\longrightarrow} \operatorname{Ker} \varphi \text {. }
$$

Proof. The sequence is obviously exact if we replace $H^{4} F$ by $H^{4} X$, by dimension counting. The assumption on $X$ implies that the map

$$
H^{4} F \oplus C H^{1} X \otimes H^{2} F \oplus C H^{2} X \stackrel{\left(f^{*}, c l^{1} \cdot f^{*}, c l^{2}\right)}{\longrightarrow} H^{4} X
$$

is surjective, as one sees from the Hochschild-Serre spectral sequence (compare $[7$, lemma 3]). It is then clear that $\operatorname{Im}\left(H^{4} X \rightarrow H^{0}\left(X, \mathcal{H}^{4}\right)\right)=\operatorname{Im}\left(H^{4} F \rightarrow H^{0}\left(X, \mathcal{H}^{4}\right)\right)$.

The hypothesis of lemma 5.1 is satisfied for quadrics of dimensions $>4$ and for a virtual Albert quadric (but not for any other 4-dimensional quadric).

The Hochschild-Serre spectral sequence defines a 3-step filtration on $H^{5} X$ :

$$
H^{5} X=F^{0} H^{5} \supseteq F^{1} H^{5} \supseteq F^{2} H^{5} \supseteq 0
$$

where $F^{0 / 1} H^{5}$ is a subgroup of $H^{1}\left(F, C H^{2} X_{s} / 2\right), F^{1 / 2} H^{5}$ is isomorphic to $H^{3} F \otimes$ $C H^{1} X$ and $F^{2} H^{5} X$ is isomorphic to $H^{5} F$. To compute Coker $\eta_{2}^{4}$, we shall filter $\operatorname{Ker} \varphi$ accordingly. Let us call

- $\bar{\varphi}_{1}$ the composite

$$
H^{2}\left(X, \mathcal{H}^{3}\right) \stackrel{\varphi}{\rightarrow} F^{0 / 2} H^{5}=H^{5} X / H^{5} F
$$

- $\bar{\varphi}$ the composite

$$
H^{2}\left(X, \mathcal{H}^{3}\right) \stackrel{\bar{\varphi}_{1}}{\longrightarrow} F^{0 / 2} H^{5} \rightarrow F^{0 / 1} H^{5}=H^{1}\left(F, C H^{2} X_{s} / 2\right)
$$


5.2. Lemma. Let $X$ be virtual Albert. Then there is a commutative diagram with exact row and column (defining $\left.(\operatorname{Ker} \bar{\varphi})_{1}\right)$

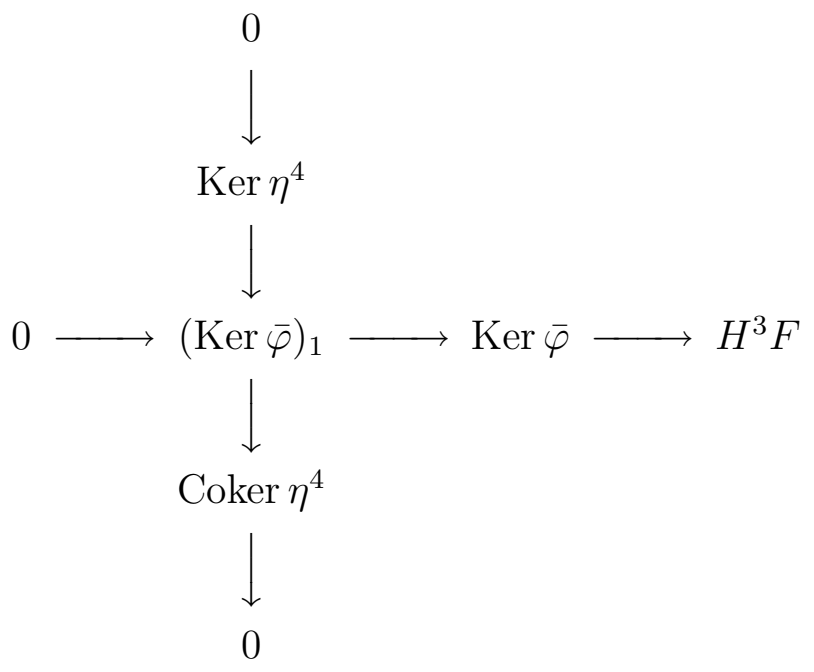

Proof. Consider the following commutative diagram:

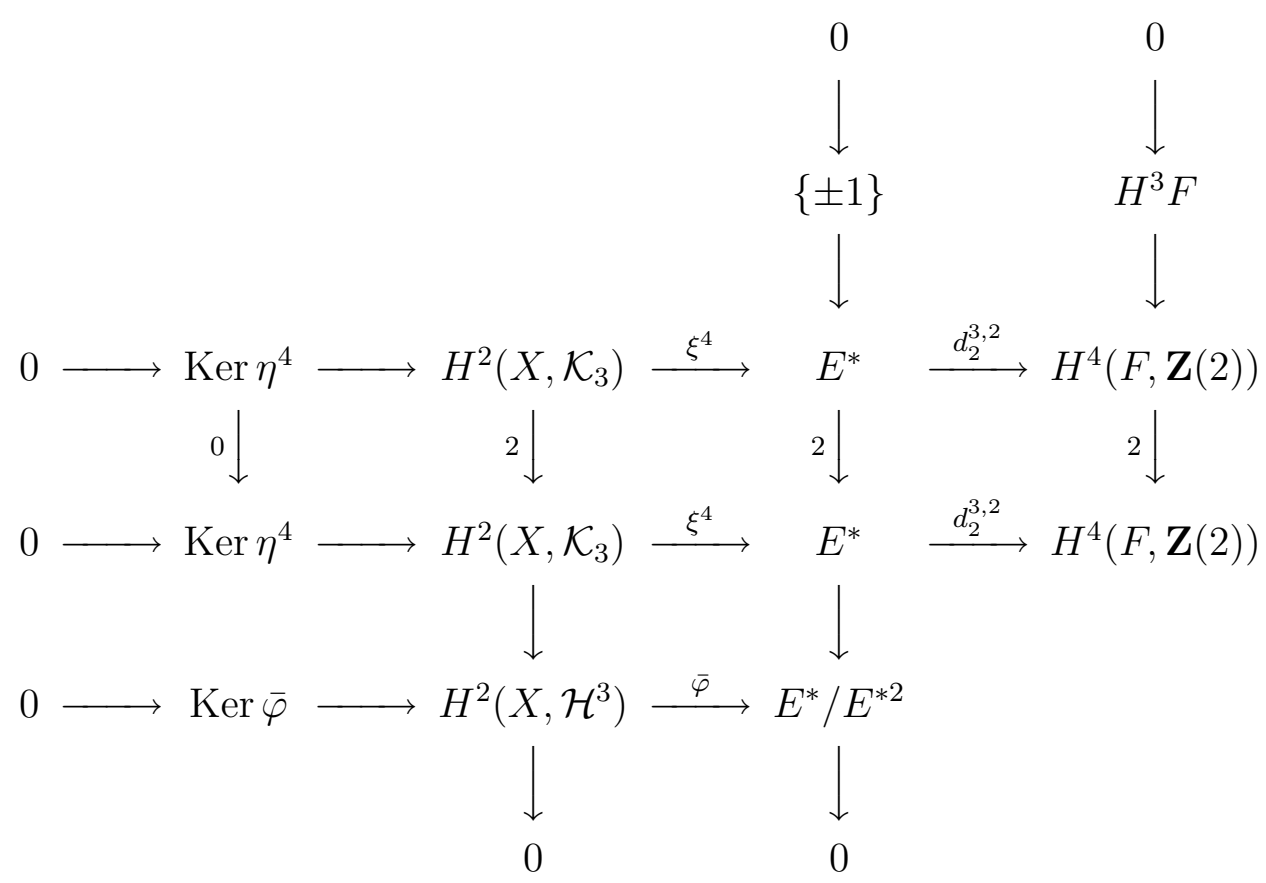

In this diagram, all columns are exact (except the left one). The rows are exact, except the first two at $E^{*}$, where their homology is Coker $\eta^{4}$ by the previous sections. A diagram chase yields a map from $\operatorname{Ker} \bar{\varphi}$ to $H^{3} F$; it is well-defined because $-1 \in \operatorname{Im} \xi^{4}$ by proposition 4.1 . For the same reason, the map $(\operatorname{Ker} \varphi)_{1} \rightarrow$ Coker $\eta^{4}$ induced by another diagram chase is well-defined; it is surjective because Coker $\eta^{4}$ has exponent 2. A last diagram chase gives an exact sequence

$$
\{ \pm 1\} \rightarrow \operatorname{Ker} \eta^{4} \rightarrow(\operatorname{Ker} \bar{\varphi})_{1}
$$


But the left map is 0: indeed, there is an element of order 2 in $H^{2}\left(X, \mathcal{K}_{3}\right)$ mapping to -1 via $\xi^{4}$. To see this, go back to the proof of proposition 4.1 and observe that $\Phi(-1,1,1)=-1$.

5.3. Lemma. The sequence

$$
H^{5}(X, \mathbf{Z}(3)) \oplus H^{5}(F, \mathbf{Z}(3)) \stackrel{\left(\underset{\pi^{*}}{\longrightarrow}\right)}{\longrightarrow} H^{5}(X, \mathbf{Z}(3)) \rightarrow H^{5} X / H^{5} F
$$

is exact.

Proof. We have the following commutative diagram with exact rows:

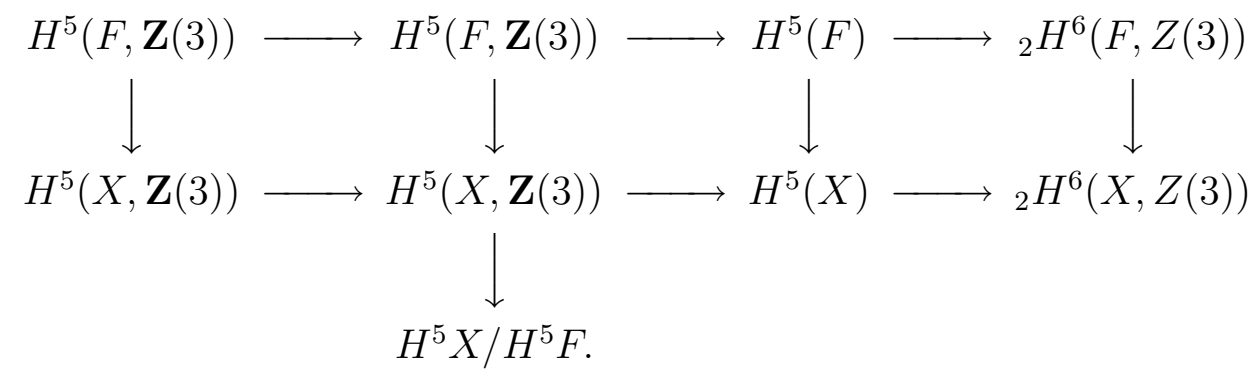

All vertical arrows except the third from the left are injective by corollary 4.20. A diagram chase now completes the proof of the lemma.

5.4. Lemma. With assumptions and notation as in lemma 5.2, we have $(\operatorname{Ker} \bar{\varphi})_{1}=$ $\operatorname{Ker} \bar{\varphi}_{1}$.

Proof. The Hochschild-Serre spectral sequence gives an exact sequence

$$
0 \rightarrow H^{3}\left(F, C H^{1}(\bar{X} \otimes \mathbf{Z} / 2)\right) \rightarrow H^{5}(X) / H^{5}(F) \rightarrow E^{*} / E^{* 2} \rightarrow 0 .
$$

As $\bar{\varphi}$ factors as $H^{2}\left(X, \mathcal{H}^{3}\right) \rightarrow H^{5} X / H^{5} F \rightarrow E^{*} / E^{* 2}$, the above exact sequence gives another map

$$
\operatorname{Ker} \bar{\varphi} \rightarrow H^{3}\left(F, C H^{1}(\bar{X} \otimes \mathbf{Z} / 2)\right) \simeq H^{3}(F)
$$

whose kernel is $\operatorname{Ker} \bar{\varphi}_{1}$. Consider the commutative diagram

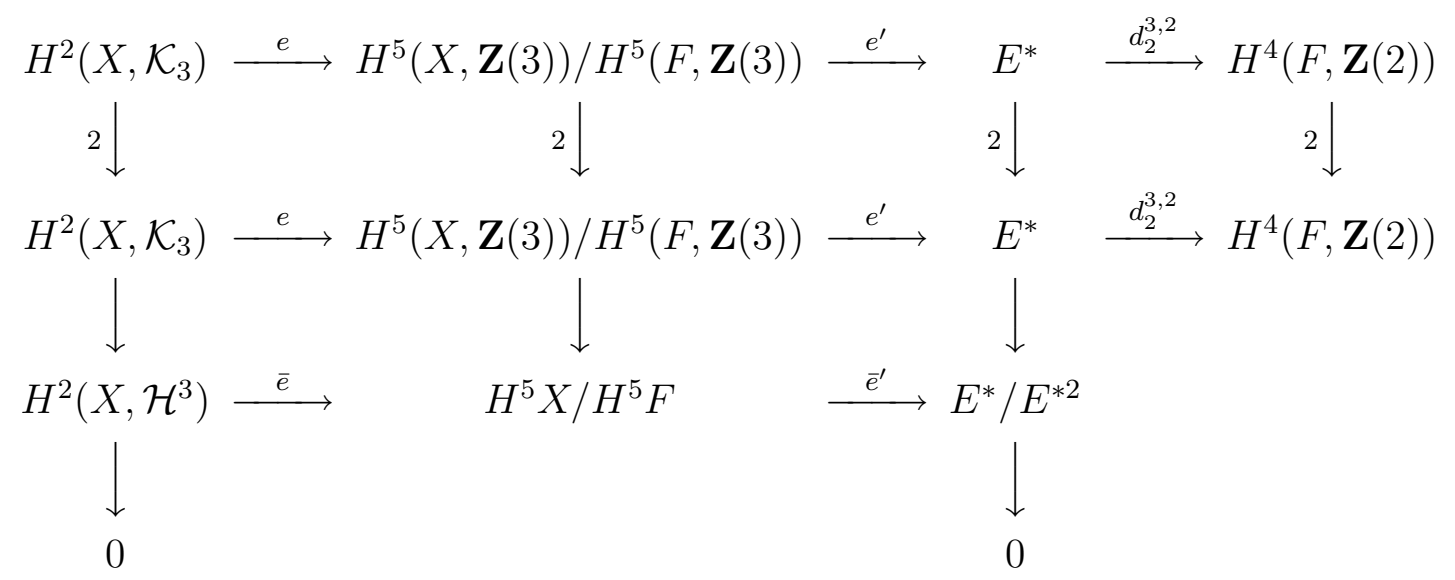

To understand the proof, the reader should compare this diagram with the one used in the proof of lemma 5.2. In the present diagram, $e$ is induced by an edge map from the coniveau spectral sequence and $e^{\prime}$ is an edge map from the motivic 
spectral sequence, so that $\xi^{4}=e^{\prime} \circ e$ (see $[9,5.4]$ and the diagram in the proof of lemma 5.2). Similarly, we have $\bar{e}^{\prime} \circ \bar{e}=\bar{\varphi}$. The top two rows are exact at $E^{*}$ and the third column from the left is exact. Moreover, the second column from the left is also exact by lemma 5.3. Finally, $e^{\prime}$ is injective by $[9,5.4]$. We need to show that, if $x \in H^{2}\left(X, \mathcal{H}^{3}\right)$ is such that $\bar{\varphi}(x)=0$ and furthermore maps to 0 in $H^{3} F$ by the diagram chase of lemma 5.2 , then $\bar{e}(x)=0$ and conversely. This follows from a straightforward diagram chase.

The group $\operatorname{Ker} \bar{\varphi}_{1}$ maps to $H^{5} F$ and we have

5.5. Lemma. The composition

$$
\text { Ker } \eta^{4} \rightarrow \operatorname{Ker} \bar{\varphi}_{1} \rightarrow H^{5} F
$$

is given by cup-product by $(-1)$.

Proof. Looking at the definition of the map $\operatorname{Ker} \eta^{4} \rightarrow H^{2}\left(X, \mathcal{K}_{3}\right)$ stemming from the diagram in $[9,5.4]$, we see that the above composition coincides with the composition of the middle row in the following commutative diagram:

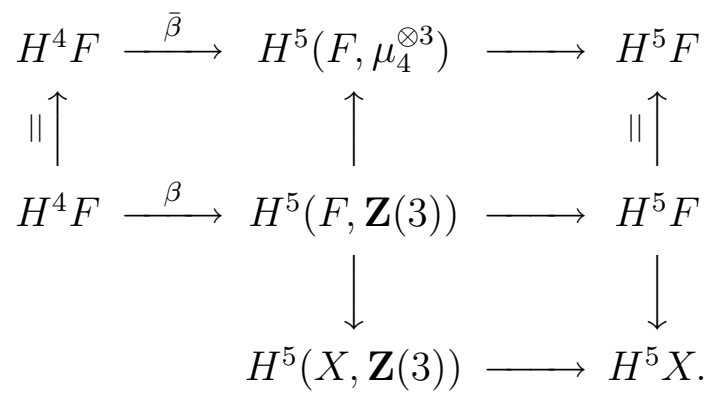

The top composition is cup-product by $(-1)$ by the Milnor conjecture and $[6$, lemma 1].

From the coniveau spectral sequence, the composition

$$
H^{2}\left(X, \mathcal{H}^{3}\right) \rightarrow H^{5} X \rightarrow H^{0}\left(X, \mathcal{H}^{5}\right)
$$

is evidently 0: therefore the image of the composition $\operatorname{Ker} \bar{\varphi}_{1} \rightarrow H^{5} F$ is contained in $\operatorname{Ker} \eta^{5}$. Moreover, the spectral sequence now gives an exact sequence

$\operatorname{Ker} \bar{\varphi}_{1} \rightarrow \operatorname{Ker} \eta^{5} \stackrel{\varepsilon}{\rightarrow} H^{1}\left(X, \mathcal{H}^{4}\right)$ 
Taking the previous lemmas into account, we therefore get a cross of exact sequences

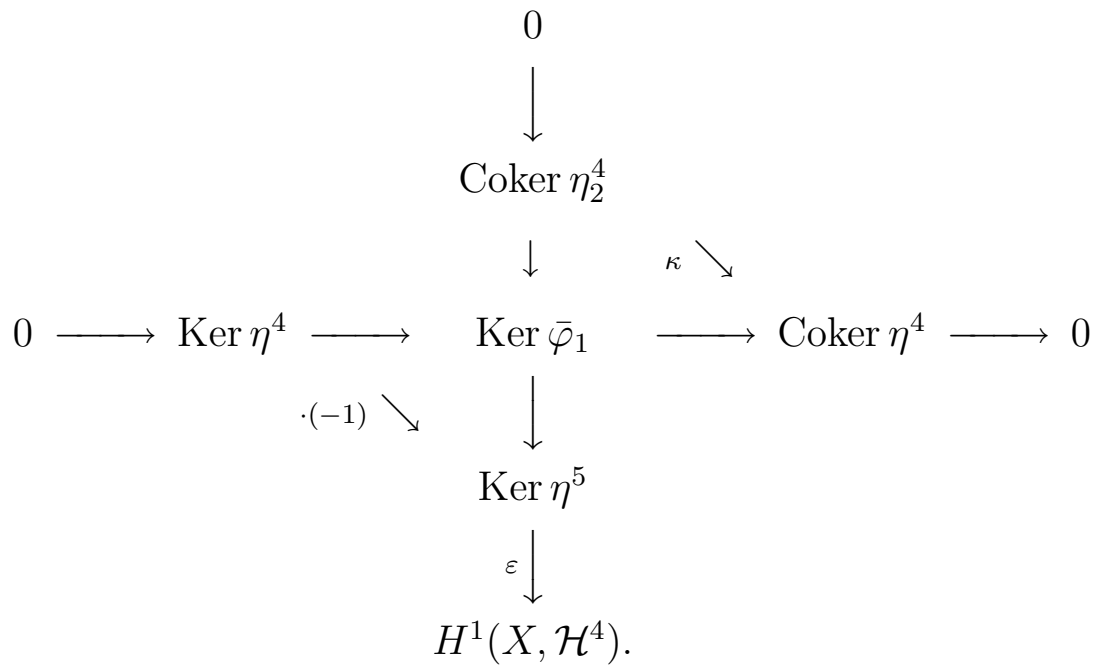

This diagram and the lemma of the 700th [21] gives back the exact sequence (1) plus an isomorphism

$$
\text { Coker } \kappa \simeq \operatorname{Ker} \varepsilon /(-1) \cdot \operatorname{Ker} \eta^{4}
$$

Appendix A. A spectral Sequence for the Étale motivic Cohomology OF AN AFFINE QUADRIC

In this appendix, we prove proposition 4.14. Recall that

$$
\tilde{\mathbf{Z}}:=f_{*} \mathbf{Z} / \mathbf{Z}
$$

where $f$ is the projection Spec $E \rightarrow \operatorname{Spec} F$. Arguing as in [9], it suffices to show:

A.1. Proposition. Let $M(U)$ be the motive of $U$, viewed in the category $D M_{-, \text {ét }}^{\text {eff }}(F)$. Then

$$
M(U) \simeq \mathbf{Z} \oplus \tilde{\mathbf{Z}}(2)[4]
$$

where $\tilde{\mathbf{Z}}(1)=\tilde{\mathbf{Z}} \otimes \mathbf{Z}(1)$.

Proof. There is an exact triangle

$$
M(U) \rightarrow M(X) \rightarrow M(Z)(1)[2] \rightarrow M(U)[1] .
$$

By $[9$, cor. 3.6], we have

$$
\begin{aligned}
& M(X) \simeq \coprod_{p \geq 0} C H^{p}\left(X_{s}\right)^{*} \otimes \mathbf{Z}(p)[2 p] \\
& M(Z) \simeq \coprod_{p \geq 0} C H^{p}\left(Z_{s}\right)^{*} \otimes \mathbf{Z}(p)[2 p]
\end{aligned}
$$

where ${ }^{*}$ denotes $\mathbf{Z}$-dual. Consequently, the above exact triangle decomposes as a direct sum of exact triangles (for $p \geq 0$ ):

$$
M^{(p)}(U) \rightarrow C H^{p}\left(X_{s}\right)^{*} \otimes \mathbf{Z}(p)[2 p] \rightarrow C H^{p-1}\left(Z_{s}\right)^{*} \otimes \mathbf{Z}(p)[2 p] \rightarrow M^{(p)}(U)[1] .
$$


By lemma 4.13, these triangles identify $M^{(p)}(U)$ to

- $\mathbf{Z}$ for $p=0$

- $\tilde{\mathbf{Z}}^{*} \otimes \mathbf{Z}(2)[4] \simeq \tilde{\mathbf{Z}}(2)[4]$ for $p=2$

- 0 otherwise.

Proposition A.1 follows.

\section{REFERENCES}

[1] J.Kr. Arason, Cohomologische Invarianten quadratischer Formen, J. Alg. 36 (1975), 448-491.

[2] H. Bass, J. Tate The Milnor ring of a global field, Lect. Notes in Math. 342, Springer, 1973, $349-446$.

[3] J.-L. Colliot-Thélène, A. Skorobogatov Groupe de Chow des zéro-cycles sur les fibrés en quadriques, K-theory 7 (1993), 477-500.

[4] R.W. Fitzgerald Witt kernels of function field extensions, Pacific J. Math. 109 (1983), 89-106.

[5] W. Jacob, M. Rost Degree four cohomological invariants for quadratic forms, Invent. Math. 96 (1989), 551-570.

[6] B. Kahn On the Scharlau transfer, Rocky Mountain J. Math. 19 (1989), 741-747.

[7] B. Kahn Lower $\mathcal{H}$-cohomology of higher-dimensional quadrics, Arch. Math. (Basel) 65 (1995), $244-250$.

[8] B. Kahn Applications of weight-two motivic cohomology, Doc. Math. 1 (1996), 395-416.

[9] B. Kahn Motivic cohomology of smooth geometrically cellular varieties, to appear in the proceedings of the 1997 Seattle algebraic $K$-theory conference, Proc. Symp. Pure Math., AMS.

[10] B. Kahn, M. Rost, R. Sujatha Unramified cohomology of quadrics, I, Amer. J. Math. 120 (1998), 841-891.

[11] B. Kahn, M. Rost, R. Sujatha Unramified cohomology of quadrics, III, in preparation.

[12] N. Karpenko Algebro-geometric invariants of quadratic forms (in Russian), Algebra-i-Analiz 2 (1990), 141-162, 262. English translation: Leningrad Math. J. 2 (1991), 119-138.

[13] M.-A. Knus, A. Merkurjev, M. Rost, J.-P. Tignol The Book of Involutions, AMS Colloquium Publications 44, AMS, 1998.

[14] T.Y. Lam The algebraic theory of quadratic forms (2nd edition), Benjamin, 1980.

[15] A.S. Merkurjev On the norm residue symbol of degree 2, Dokl. Akad. Nauk SSSR 261 (1981), 542-547. English translation: Soviet Math. Dokl. 24 (1981), 546-551.

[16] A.S. Merkurjev The group $S K_{2}$ for quaternion algebras, Izv. Akad. Nauk SSSR 52 (1988), 310-335. English translation: Math. USSR Izv. 32 (1989), 313-337.

[17] A.S. Merkurjev K-theory of simple algebras, in: W. Jacob and A. Rosenberg (ed.), $K$-theory and algebraic geometry: connections with quadratic forms and division algebras, Proceedings of Symposia in Pure Mathematics 58 (I) (1995), 65-83.

[18] A.S. Merkurjev The norm residue homomorphism for fields, Mathematics in St. Petersburg, AMS Trans. 2, 174, AMS, Providence, 1996, 49-71.

[19] A.S. Merkurjev R-equivalence and rationality problem for semi-simple adjoint classical algebraic groups, Publ. Math. IHES. 84 (1996), 189-213.

[20] A.S. Merkurjev, A.A. Suslin The norm residue homomorphism of degree three (in Russian), Izv. Akad. Math. SSSR. Engl. translation: Math. USSR Izv. 36 (1991), 349-367.

[21] A.S. Merkurjev, J.-P. Tignol Galois cohomology of biquadratic extensions, Comment. Math. Helv. 68 (1993), 138-169.

[22] A. Suslin Torsion in $K_{2}$ of fields, K-theory 1 (1987), 5-29.

[23] M. Szyjewski The fifth invariant of quadratic forms (in Russian), Algebra Anal. 2 (1990), 213-234. English translation: Leningrad Math. J. 2 (1991), 179-198.

[24] J.-P. Tignol Corps à involution neutralisés par une extension abélienne élémentaire, Lect. Notes in Math. 844 (1981), 1-34.

[25] V. Voevodsky The Milnor conjecture, preprint, 1996. 
[26] E. Witt Über ein Gegenbeispiel zum Normensatz, Math. Zeit. 39 (1935), 462-467.

Institut de Mathématiques de Jussieu, Equipe ThÉories GÉométriques, Université Paris 7, Case 7012, 75251 Paris Cedex 05, France

E-mail address: kahn@math.jussieu.fr

School of Mathematics, Tata Institute of Fundamental Research, Homi Bhabha RoAD, BOMBAY 400005, IndiA

E-mail address: sujatha@math.tifr.res.in 